\title{
Curcumin: Novel Treatment in Neonatal Hypoxic-Ischemic Brain Injury
}

\author{
Eridan Rocha-Ferreira ${ }^{1,2 \dagger}$, Claudia Sisa ${ }^{1 \dagger}$, Sarah Bright ${ }^{1}$, Tessa Fautz ${ }^{1}$, Michael Harris', \\ Ingrid Contreras Riquelme ${ }^{1}$, Chinedu Agwu', Tugce Kurulday ${ }^{1,3}$, Beenaben Mistry ${ }^{1}$, \\ Daniel Hill',4, Sigrun Lange ${ }^{5}$ and Mariya Hristova ${ }^{1 *}$

\begin{abstract}
'Department of Maternal and Fetal Medicine, Perinatal Brain Repair Group, UCL Institute for Women's Health, London, United Kingdom, ${ }^{2}$ Department of Neuroscience and Physiology, Sahlgrenska Academy, University of Gothenburg, Gothenburg, Sweden, ${ }^{3}$ Department of Molecular Biology and Genetics, Izmir Institute of Technology, Izmir, Turkey, ${ }^{4}$ Department of Visual Neuroscience, Glaucoma and Retinal Neurodegeneration Group, UCL Institute of Ophthalmology, London, United Kingdom, ${ }^{5}$ School of Life Sciences, Tissue Architecture and Regeneration Research Group, University of Westminster, London, United Kingdom
\end{abstract}

Hypoxic-ischemic encephalopathy $(\mathrm{HIE})$ is a major cause of mortality and morbidity in neonates, with an estimated global incidence of 3/1,000 live births. HIE brain damage is associated with an inflammatory response and oxidative stress, resulting in the activation of cell death pathways. At present, therapeutic hypothermia is the only clinically approved treatment available for HIE. This approach, however, is only partially effective. Therefore, there is an unmet clinical need for the development of novel therapeutic interventions for the treatment of HIE. Curcumin is an antioxidant reactive oxygen species scavenger, with reported anti-tumor and anti-inflammatory activity. Curcumin has been shown to attenuate mitochondrial dysfunction, stabilize the cell membrane, stimulate proliferation, and reduce injury severity in adult models of spinal cord injury, cancer, and cardiovascular disease. The role of curcumin in neonatal HIE has not been widely studied due to its low bioavailability and limited aqueous solubility. The aim of this study was to investigate the effect of curcumin treatment in neonatal HIE, including time of administration and dose-dependent effects. Our results indicate that curcumin administration prior to HIE in neonatal mice elevated cell and tissue loss, as well as glial activation compared to $\mathrm{HI}$ alone. However, immediate post-treatment with curcumin was significantly neuroprotective, reducing grey and white matter tissue loss, TUNEL + cell death, microglia activation, reactive astrogliosis, and iNOS oxidative stress when compared to vehicle-treated littermates. This effect was dose-dependent, with $200 \mu \mathrm{g} / \mathrm{g}$ body weight as the optimal dose-regimen, and was maintained when curcumin treatment was delayed by 60 or 120 min post-HI. Cell proliferation measurements showed no changes between curcumin and $\mathrm{HI}$ alone, suggesting that the protective effects of curcumin on the neonatal brain following $\mathrm{HI}$ are most likely due to curcumin's anti-inflammatory and antioxidant properties, as seen in the reduced glial and iNOS activity. In conclusion, this study suggests curcumin as a potent neuroprotective agent with potential for the treatment of HIE. The delayed application of curcumin further increases its clinical relevance.

Keywords: curcumin, hypoxia, ischemia, neuroprotection, neonate, oxidative stress 


\section{INTRODUCTION}

Neonatal hypoxic-ischemic (HI) brain injury has an incidence of 1-3 per 1,000 live births (Rocha-Ferreira and Hristova, 2016), and results in almost 1 million neonatal deaths worldwide (Lawn et al., 2005; Rocha-Ferreira and Hristova, 2016). Approximately, $30 \%$ of $\mathrm{HI}$ cases will develop lifelong disabilities, including cerebral palsy, seizures, and cognitive impairments (RochaFerreira and Hristova, 2016; Lundgren et al., 2018). The severity of such disabilities depends on the stage of gestation at which the HI event occurs and its duration (Sanders et al., 2010).

The pathology of HI brain damage is characterized by an initial primary energy loss phase, where oxygen and glucose deprivation in the cell causes a drop in the mitochondrial oxidative phosphorylation, resulting in reduced adenosine triphosphate (ATP) availability, triggering excitotoxicity (RochaFerreira and Hristova, 2016), neurotoxicity (Sanders et al., 2010), and oxidative stress (Hope et al., 1984; Penrice et al., 1997). Early after the HI insult, damaged neuronal cells stimulate a pro-inflammatory immune response where activated microglia produce cytokines such as IL-1 $\beta$ and TNF $\alpha$, proteases, and complement factors (Rocha-Ferreira and Hristova, 2016).

After successful resuscitation, a short latent/recovery period occurs. However, during reperfusion, the majority of the oxidative markers are produced, and in cases of an initial prolonged $\mathrm{HI}$ insult, the primary energy failure cannot be compensated, therefore, leading to a secondary energy drop (Rocha-Ferreira and Hristova, 2016). Inflammatory processes and continued excitotoxicity lead to an impaired equilibrium between pro- and anti-inflammatory cytokines, as well as significant damage of the mitochondria machinery (Peng and Greenamyre, 1998; PukaSundvall et al., 2000). This is associated with increased levels of hydrogen peroxide $\left(\mathrm{H}_{2} \mathrm{O}_{2}\right)$ and nitrogen oxide (NO), overproduction of free radicals, and reactive oxygen species (ROS) which, together with the persistent inflammation, stimulate necrosis (Li et al., 1998), apoptosis (Johnston et al., 2002), and autophagy (Rocha-Ferreira and Hristova, 2016) cell death pathways.

Therapeutic hypothermia $(\mathrm{TH})$ is the standard clinical treatment applied in moderate to severe injury; however, it does not guarantee total recovery of the treated neonates with effectiveness of only $55 \%$ of cases and the remaining infants still develop neurological deficits (Gluckman et al., 2005). Thus, further studies on improving $\mathrm{TH}$ success rate and finding therapeutic alternatives are urgently required.

Curcumin, the major phytochemical component of the plant Curcuma longa, is extracted from its rhizomes (turmeric), and regularly consumed in South Asian diets (Shishodia et al., 2005; Pescosolido et al., 2013). Except for turmeric usage as dietary spice and coloring agent, curcumin has been studied for its therapeutic role in several pathological conditions; its effects have been investigated in cancer (Naksuriya et al., 2014; Ahmad et al., 2016), inflammation (Kim et al., 2003; Sandur et al., 2007), infections, cardiovascular diseases (Nishiyama et al., 2005; Liu and Hong, 2006), fibrosis, and neurological disorders (Spagnuolo et al., 2016). Such pharmacological success relies on the phytochemical abilities of acting on many critical pathways, showing anti-inflammatory (Tham et al., 2010), anti-oxidant
(Alexandrow et al., 2012), anti-microbial (Moghadamtousi et al., 2014), and anti-apoptotic (Yu et al., 2010; Jaisin et al., 2011) effects, as well as capability to promote stem cell differentiation (Gu et al., 2012; Mujoo et al., 2012; Chen et al., 2014). Effects of curcumin on mitochondrial function have also been reported (Bavarsad et al., 2018; He et al., 2018; Hsiao et al., 2018; Momekova et al., 2018; Naserzadeh et al., 2018) and furthermore, hormetic effects of curcumin are receiving increased attention (Moghaddam et al., 2018). Curcumin pleiotropic activity is due to its chemical structure, and specifically to the o-methoxy phenolic groups (Priyadarsini and Indira, 2014), which are central for chemical reactions. In fact, curcumin scavenging abilities on ROS and free radicals (Trujillo et al., 2014) seem to rely on this functional group, as well as its modulatory role on pro-inflammatory cytokines [tumor necrosis factor alpha (TNF $\alpha)$, interleukin (IL)1 (Alexandrow et al., 2012), and IL6 (Maheshwari et al., 2006)] and its inhibition of signal transducer and activator of transcription 3 (STAT3) phosphorylation (Maheshwari et al., 2006; Alexandrow et al., 2012). As STAT3 is crucial for astrocyte differentiation (Hong and Song, 2014), its inhibition can reduce reactive astrogliosis post-HI. Clinical trials have demonstrated remarkable safety profile and good tolerance for curcumin in humans at doses of $8 \mathrm{~g} / \mathrm{day}$ (Dhillon et al., 2008). Moreover, the small molecular weight $(368.385 \mathrm{~g} / \mathrm{mol})$, and dimensions allow it to cross the blood-barrier (BBB) (Priyadarsini and Indira, 2014), increase researchers interest for curcumin application in neurodegenerative disorders including traumatic brain injury (Wu et al., 2006), Alzheimer's (Ray et al., 2011; Mutsuga et al., 2012), and Parkinson's (Mythri et al., 2011) diseases.

The pathways on which curcumin acts, overlap with those activated after HI injury, including oxidative stress and inflammation. So far curcumin has been vaguely tested in neonatal $\mathrm{HI}$ injury in rats, either through oral administration (Cui et al., 2017) or through intraperitoneal single dose delivery of nanoparticle- or DMSO-dissolved curcumin (Joseph et al., 2018). Both studies report attenuation of damage; however, there is no systematic approach to dose response or long-term protection. In fact, Joseph et al. report no effect of the DMSO-dissolved curcumin suggesting that it is only the nanoparticle-loaded curcumin showing protection (Joseph et al., 2018). The aim of our study was to assess the short- and long term-effects of curcumin administered immediately after neonatal HI insult, to determine the lowest neuroprotective dose of the compound and whether delayed administration at 60 and $120 \mathrm{~min}$ post-HI would show neuroprotection. Our observations show that immediate or delayed administration of curcumin strongly protects the neonatal brain following $\mathrm{HI}$ insult. These protective effects are though not a consequence of changes in cell proliferation, but possibly related to alterations in STAT3 phosphorylation and prohibitin (PHB) protein levels. The STAT3 Y705 phosphorylation site is responsible for the transcription properties of STAT3 and previous results from our group showed bilateral upregulation and involvement of STAT3 Y705 in neonatal HI brain damage (Hristova et al., 2016). The STAT3 S727 phosphorylation site is downstream of extracellular signal-regulated kinase (ERK), respectively of RAS and is responsible for mitochondrial survival in cancer studies (Gough et al., 2009). 
PHB is a pleiotropic multifaceted protein and an essential factor in mitochondrial homeostasis, biogenesis, degradation, and response to stress (Peng et al., 2015; Ande et al., 2017; Hernando-Rodríguez and Artal-Sanz, 2018). In mitochondria, $\mathrm{PHB}$ acts as a scaffold protein and is thus crucial for regulation of mitochondrial architecture, mitochondrial dynamics, morphology, and biogenesis, and furthermore stabilizes the mitochondrial genome (Merkwirth et al., 2012; Peng et al., 2015).

Our data demonstrate that the neuroprotective effects of curcumin are likely the consequence of changes in the levels of PHB and of STAT3 phosphorylation (Y705 and S727); thus affecting inflammation and mitochondrial dysfunction post-HI. Therefore, curcumin could be considered a potent candidate for neuroprotective treatment in neonatal HI brain damage.

\section{MATERIALS AND METHODS}

\section{HI Insult}

All animal experiments and care protocols were carried out according to the UK Animals (Scientific Procedures) Act 1986 and approved by the Home Office (PPL70/8784). The ARRIVE guidelines were followed. All experiments involved postnatal day 7 C57/B16 mice (P7) bred in house.

The surgical procedures were performed as previously described (Hristova et al., 2010; Kendall et al., 2012; Lange et al., 2014; Rocha-Ferreira et al., 2015). Briefly, male and female P7 mice were anesthetized with isoflurane (5\% induction and $1.5 \%$ maintenance). The left common carotid artery was permanently occluded with $8 / 0$ polypropylene suture and the wound closed with tissue glue. The mice recovered at $36^{\circ} \mathrm{C}$ and were returned to the dam for $2 \mathrm{~h}$. The pups were then placed in a hypoxia chamber and exposed to humidified $8 \%$ oxygen/92\% nitrogen ( $3 \mathrm{~L} / \mathrm{min}$ ) at $36^{\circ} \mathrm{C}$ for $60 \mathrm{~min}$, resulting in moderate to severe brain damage (Lange et al., 2014; Hristova et al., 2016).

The P7 rodent HI model, though slightly preterm, presents phenotypical similarities to the grey and white matter injury observed in humans, i.e., tissue loss, cell-death, microgliamediated immune response, and astrogliosis as well as alteration in neurobehavioral performance (Vannucci and Vannucci, 1997).

\section{Pharmacological Treatment}

Curcumin (LKT Laboratories, UK) was dissolved in $100 \%$ DMSO to concentrations of 20,44, 100, 200, and $400 \mathrm{ug} /$ ul. The animals were injected $20 \mathrm{~min}$ before, immediately after, or after a 60 or $120 \mathrm{~min}$ delay following a $60 \mathrm{~min} \mathrm{HI}$ insult. The animals received a single intraperitoneal injection of $0.5 \mathrm{ul} / \mathrm{g}$ body weight (BW) resulting in doses of 10,22 , 50,100 , or $200 \mu \mathrm{g} / \mathrm{g}$, respectively, based on previous studies (Shukla et al., 2008; Zhao et al., 2010).

\section{Tissue Sample Preparation}

The animals were sacrificed at $48 \mathrm{~h}$ or at 21 days post-HI by intraperitoneal injection of pentobarbitone and perfused with $30 \mathrm{ml}$ (for $48 \mathrm{~h}$ ) or $90 \mathrm{ml}$ (for 21 days) $4 \%$ paraformaldehyde in $0.1 \mathrm{M}$ phosphate buffer $(\mathrm{PB})$. The brains were removed, post fixed in $4 \%$ paraformaldehyde/0.1 $\mathrm{M} \mathrm{PB}$ for $1 \mathrm{~h}$ at $4^{\circ} \mathrm{C}$, and cryoprotected in 30\% sucrose/PB solution for $24 \mathrm{~h}$ as previously described (Rocha-Ferreira et al., 2015; Hristova et al., 2016). The brains were then frozen on dry ice, cut on a cryostat into sequential $40 \mu \mathrm{m}$ sections, and stored at $-80^{\circ} \mathrm{C}$ until used.

\section{Immunohistochemistry and Histological Analysis}

Five sections from each brain $(400 \mu \mathrm{m}$ apart) were rehydrated in distilled water and stained using immunohistochemistry as previously described (Möller et al., 1996). Briefly, the sections were incubated overnight with rat anti-CD11b aM integrin subunit $(1: 5,000$, Serotec, UK), rabbit polyclonal anti-GFAP (1:6,000, DAKO, UK), rabbit anti-iNOS (1:500, Santa Cruz, USA), polyclonal rat anti-BrdU (1:400, Abcam, UK), or rabbit anti-myelin basic protein (MBP) (1:200, Abcam, Cambridge, $\mathrm{UK}$ ) primary antibodies, for $1 \mathrm{~h}$ with biotinylated goat antirabbit or -rat (1:100, Vector, UK) secondary antibodies, followed by incubation with Avidin-Biotinylated horseradish peroxidase Complex (Vector, UK) and visualization with diaminobenzidine/ $\mathrm{H}_{2} \mathrm{O}_{2}$ (Fisher Scientific, UK). The visualization for BrdU antibody required $\mathrm{Co} / \mathrm{Ni}$ enhancement.

Five further sections from each brain with the same spacing were stained using Terminal transferase mediated d-UTP nick end labeling (TUNEL) (Roche, UK). The staining procedure followed the manufacturer protocol with $\mathrm{Co} / \mathrm{Ni}$ enhancement.

Five more sections per brain with the same spacing were stained with Cresyl-Violet (Nissl).

To detect and identify the different types of proliferating cells following neonatal $\mathrm{HI}$, we used double labeling for BrdU and: rabbit polyclonal anti-IBA1 (microglia, 1:2,000, Wako, Japan), rabbit polyclonal anti-GFAP (astroglia, 1:6,000, DAKO, $\mathrm{UK}$ ), guinea pig polyclonal anti-NG2 (oligodendrocyte precursors, 1:400, Bill Stallcup, USA), or mouse monoclonal anti-NeuN (neurons, 1:25,000, Millipore, UK). The protocol was performed as previously described (Hristova et al., 2010). The sections were covered with VectaShield (Vector) and stored in the dark at $4^{\circ} \mathrm{C}$ before use. The number of BrdU positive and the double positive cells was counted in three fields at $\times 20$ magnification. The percentage of the double positive cells as a fraction of the overall number was calculated and presented.

\section{AlphaM Score}

Immunohistochemistry for $\mathrm{\alpha M}$ integrin as an early microglial activation marker (Raivich et al., 1999; Hristova et al., 2010; Kendall et al., 2012; Lange et al., 2014; Rocha-Ferreira et al., 2015), was performed as previously described (Rocha-Ferreira et al., 2015; Hristova et al., 2016). Semi-quantitative scores were allocated to each brain region (cortex, pyriform cortex, hippocampus, striatum, thalamus, and external capsule) by two independent observers blinded to the treatment of the groups.

\section{TUNEL, BrdU, and iNOS}

TUNEL positive cell death or iNOS and BrdU positive cells were assessed at $48 \mathrm{~h}$ following $\mathrm{HI}$ through bilateral counting of the number of positive cells in three different optical fields at $\times 20$ magnification. Cortex, pyriform cortex, hippocampus, 
striatum, thalamus, and external capsule were assessed for TUNEL and BrdU. Due to the exclusive expression of iNOS in hippocampus, this marker was assessed only in that region. The counts were averaged per animal and per group.

\section{Optical Luminosity}

The intensity of the GFAP or MBP staining in the tissue was assessed using optical luminosity values (Rocha-Ferreira et al., 2015; Hristova et al., 2016). Images for ipsilateral and contralateral sides were captured with a Sony AVT-Horn 3CCD color video camera (24 bit RGB, $760 \times 570$ pixel resolution) in three different optical fields in the regions of interest. We used Optimas 6.5 software to obtain the mean and standard deviation (SD) for optical luminosity values (OLV). SD was subtracted from the mean for each image and the resulting value was subtracted from the values acquired for the surrounding glass (Möller et al., 1996). OLVs for GFAP assessment were evaluated in cortex, pyriform cortex, hippocampus, striatum, thalamus and external capsule, while for MBP assessment OLVs were recorded in external capsule and striatum.

\section{Infarct Volume Measurement}

The Cresyl-Violet stained sections were scanned and imported into Optimas 6.5 image analysis software. The areas of intact staining in the cortex, pyriform cortex, hippocampus, striatum, thalamus, and external capsule were outlined and bilaterally measured using Fiji Image $\mathrm{J}$ (NIH, USA). In the long-term assessment experiments the intact staining of the different regions as well as the whole hemisphere were outlined and bilaterally measured. The percentage tissue loss was then calculated by converting the measured injured and uninjured areas into square millimeters and then transformed to a volume through multiplication by $400 \mu \mathrm{m}$. The sum of these volumes was then used to calculate the percentage of surviving brain tissue as ipsilateral/contralateral $\times 100$ (Kendall et al., 2006).

\section{Behavioral Assessment}

The slipping test was performed at 21 days (P28) post-HI. It is a modification of the balance beam test (Luong et al., 2011) used for evaluation of motor balance and co-ordination. This test detects motor deficits resulting from central nervous system lesions, as well as aging and genetic and pharmacological interventions (Luong et al., 2011). The apparatus consists of a metal grid $50 \mathrm{~cm}$ in length, placed about $20 \mathrm{~cm}$ above a table top between the housing cage and a new clean cage. The starting point is the clean cage and the finish consisted of the housing nesting cage. The animals were allowed to walk freely on the grid for $1 \mathrm{~min}$. The task was recorded, and the videos were reviewed by two team members blinded to the mouse groups. The number of missed steps was counted and presented as a percentage of the total number of steps for each animal. The results were then averaged per group.

At P7, the levels of testosterone in male and female mice do not differ (Clarkson and Herbison, 2016). However, at 21 days, testosterone levels between male and female mice are different and a lot of clinical and experimental evidence suggests important differences between males and females, with increased loss of male hippocampal volume after chronic postnatal hypoxia (Mayoral et al., 2009; Gobinath et al., 2017). Thus, the assessments for 21 days took gender into account.

\section{Western Blot Analysis}

The animals were sacrificed at $1 \mathrm{~h}$ post $\mathrm{HI}$ by intraperitoneal injection of pentobarbitone and hippocampus was extracted from treated and un-treated brains and snaps frozen before homogenization in RIPA+ buffer (Sigma, UK) containing 10\% protease inhibitor complex (Sigma, UK). The time point was chosen as coinciding with time of maximum expression of phosphorylated STAT3 Y705 (Hristova et al., 2016). Total protein was thereafter extracted from the homogenized hippocampal tissue as follows: the homogenized tissue was incubated with the RIPA+ buffer on a shaking platform on ice for $2 \mathrm{~h}$, pipetting gently with regular intervals. Thereafter, the homogenates were centrifuged at $16,000 \mathrm{~g}$ at $4^{\circ} \mathrm{C}$ for $20 \mathrm{~min}$ and the supernatant containing the extracted protein collected. The protein extracts were re-constituted in $2 \times$ Laemmli sample buffer (BioRad, UK) containing $5 \% \beta$-mercaptoethanol (Sigma, UK) and boiled for $5 \mathrm{~min}$ at $100^{\circ} \mathrm{C}$ before separation by SDS-PAGE, using 4-20\% Mini-Protean TGX protein gels (BioRad, UK), followed by Semi-Dry Western blotting analysis. Approximately, $5 \mu \mathrm{g}$ of protein was loaded per lane and even transfer to nitrocellulose membranes $(0.45 \mu \mathrm{m}$, BioRad, UK) was assessed using Ponceau $\mathrm{S}$ staining (Sigma, UK). The membranes were blocked for $1 \mathrm{~h}$ at room temperature (RT) in 5\% bovine serum albumin (BSA, Sigma, UK) in Tris-buffered saline (TBS) with $0.001 \%$ Tween 20 (TBS-T), followed by overnight incubation at $4^{\circ} \mathrm{C}$ with the following primary antibodies: anti-prohibitin (Abcam, UK), Phospho-Stat3 (Ser727) (Cell Signaling, UK and Abcam, UK), or Phospho-Stat3 (Tyr705) (Cell Signaling, UK and Abcam, UK). Thereafter, membranes were washed three times for $10 \mathrm{~min}$ in TBS-T, incubated for $1 \mathrm{~h}$ at RT with an HRP-labeled antirabbit IgG secondary antibody (BioRad, UK), followed by five TBS-T washes and one final TBS wash, and thereafter they were visualized using ECL (Amersham, UK) and the UVP BioDoc-ITTM System. HRP-conjugated anti- $\beta$-actin antibody $(1 / 5,000$ in TBS-T, Abcam, UK) was used for internal loading control and densitometry analysis was carried out using ImageJ.

\section{Statistics}

Statistical significance was assessed through repeated testing using Mixed Linear Model with SPSS 23.0 and GraphPad Prism 7.0 software, treating region as the repeated measure. For each outcome six regions of the brain were examined. It is likely that with repeated measures such as the observations from a single subject are correlated, the first stage of the analysis included the observations from all the regions tested in a single mixed model with a random subject effect, to produce an estimate of the treatment effect and associated inference that accounts for the correlations in the data arising from the repeated measures. Further post hoc Student $t$-tests were carried out to assess evidence for subregional differences, $p<0.05$. For comparison of more than two groups, we used two-way ANOVA 
with post hoc Tukey or Bonferroni tests to assess evidence for subregional differences, $p<0.05$. If parametric analysis was inappropriate, the non-parametric Kruskal-Wallis test was used, followed by Bonferroni-corrected pairwise-contrasts to investigate differences between treatment-conditions. For each outcome, the main effect from the mixed linear model or the KruskalWallis test is reported, followed by the results from the individual regional $t$-tests. In our data, a main effect is the effect of an independent variable (treatment) on a dependent variable (damage marker) averaged across the levels of any other independent variables (brain regions). All data are presented as Mean + SEM.

\section{RESULTS}

\section{Intraperitoneal Pre-treatment With Curcumin Increases the Glial Response Following $\mathrm{HI}-$ Insult in the Neonate}

To determine the biological impact of pre-treatment with curcumin, the animals were injected intraperitoneally with $100 \mu \mathrm{g} / \mathrm{g}$ BW curcumin and the effect on brain volume loss, TUNEL+ cell death, and on reactive astrogliosis and microglial activation at $48 \mathrm{~h}$ were examined following the HI-insult. As shown in Figure 1, curcumin pre-treatment had no main effect

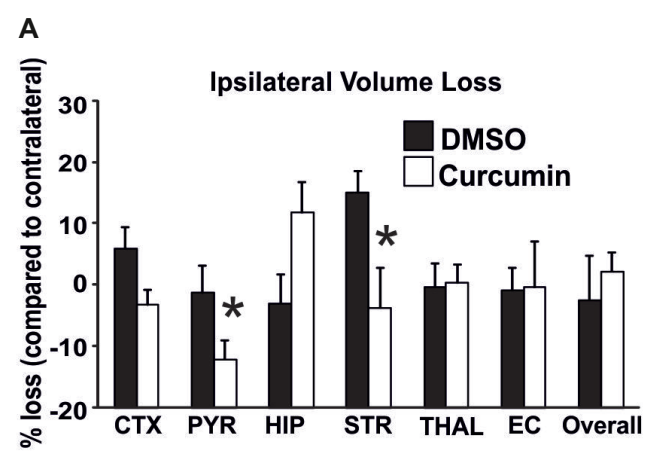

B
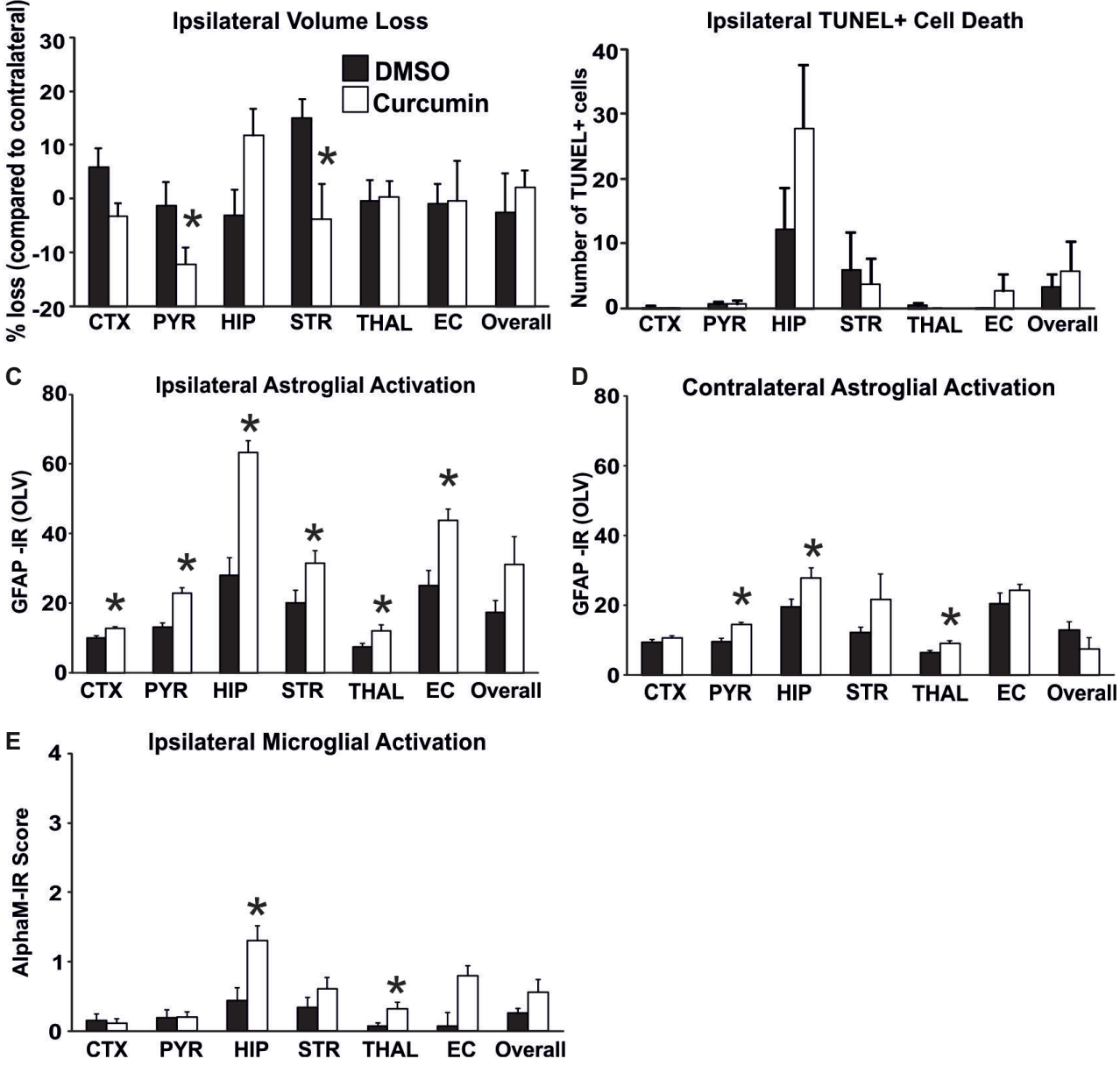

FIGURE 1 | Intraperitoneal injection of curcumin (100 $\mu \mathrm{g} / \mathrm{g}$ BW) in P7 mice 20 min before HI does not affect tissue damage and cell death, but significantly increases glial response. (A) Ipsilateral forebrain Nissl staining (Cresyl-Violet, at rostral parietal level) - Quantification of ipsilateral brain tissue volume loss of DMSO and curcumin pre-treated animals at $48 \mathrm{~h}$ following HI-insult. Curcumin pre-treatment $(n=12)$ did not affect volume loss compared to DMSO-treated littermates $(n=11)$ (Mixed Linear Model treating region as a repeated measure $p=0.156)$. However significant, individual decrease ( $t$-test) was registered in pyriform cortex $(p=0.04)$ and striatum $(p=0.02)$. (B) The number of TUNEL+ dying cells (per 20x eye-field) at $48 \mathrm{~h}$ following $\mathrm{HI}$, was not affected in the curcumin pre-treated group compared to DMSO-treated littermates (Mixed Linear Model treating region as a repeated measure $p=0.053$ ). (C,D) GFAP immunoreactivity at $48 \mathrm{~h}-\mathrm{quantification}$ of the ipsilateral (C) and contralateral (D) side in optical luminosity values (OLV, Mean + SEM). Note the increased levels of GFAP immunoreactivity in the curcumin pre-treated animals, with significant, individual increase ( $t$-test) in ipsilateral cortex $(p=0.002)$, pyriform cortex $(p=0.0001)$, hippocampus $\left(p=3 \times 10^{-5}\right)$, striatum $(p=0.038)$, thalamus $(p=0.026)$, and external capsule $(p=0.003)$, and contralateral pyriform cortex $(p=0.0002)$, hippocampus $(p=0.04)$, and thalamus $(p=0.013)$. Mixed Linear Model treating region as a repeated measure revealed $p=0.0001$ for ipsilateral and $p=0.007$ for contralateral side, respectively. (E) Activation of $\alpha \mathrm{M}+$ microglia - ipsilateral $\alpha \mathrm{M}$ microglial activation score (Mean + SEM). Pre-treatment with $100 \mu \mathrm{g} / \mathrm{g}$ BW curcumin increased $\alpha \mathrm{M}+$ microglial activation with significant, individual increase ( $t$-test) in hippocampus $(p=0.008)$ and thalamus $(p=0.03)$. Mixed Linear Model treating region as a repeated measure revealed $p=0.019$. ( $\left.{ }^{*} p<0.05\right)$. Abbreviations: CTX, cerebral cortex; EC, external capsule; HIP, hippocampus; PYR, pyriform cortex; STR, striatum; THAL, thalamus. 
on brain volume loss (Figure 1A) and TUNEL+ cell death (Figure 1B). However, individual significant attenuation of brain volume loss ( $t$-test) was observed in pyriform cortex and striatum (Figure 1A). Interestingly, an increase of tissue loss, although not significant, was observed in the hippocampus of curcumin treated animals. Pre-treatment with $100 \mu \mathrm{g} / \mathrm{g} \mathrm{BW}$ of curcumin significantly increased $\mathrm{HI}$-induced and predominantly ipsilateral reactive astrogliosis and microglial activation (Figures 1C-E). Compared to DMSO-treated littermates, the curcumin pre-treated animals revealed more GFAP-immunoreactivity (Figures $\mathbf{1 C}, \mathbf{D}$ ). Assessment across the different forebrain regions through Mixed Linear Model treating region as a repeated measure revealed a clear increase on the ipsilateral, and milder increase on the contralateral side $(p=0.0001$ and $p=0.007$, respectively), with individual significant increase of $20-50 \%$ in all studied ipsilateral regions, and of $30-40 \%$ in contralateral pyriform cortex, hippocampus and striatum ( $p<0.05$ in $t$-test). Curcumin pre-treatment also increased microglial activation score (Figure 1E) based on the $\mathrm{aM}$ integrin immunoreactivity. Regional assessment revealed an increase in activation score in the curcumin pre-treated group (Mixed Linear Model treating region as a repeated measure $p=0.019$ ) with significant increase of $30-50 \%$ in hippocampus and thalamus (Figure 1E).

\section{Immediate Post-treatment With Curcumin Reduces Brain Damage Following Neonatal HI-Insult}

Compared to DMSO-treated animals, intraperitoneal injection of $200 \mu \mathrm{g} / \mathrm{g}$ BW curcumin straight after the HI-insult significantly reduced brain damage markers (tissue loss, TUNEL+ cell death, reactive astrogliosis, and microglial activation) at $48 \mathrm{~h}$ post-HI. As shown in Figures 2A-C, curcumin post-treatment markedly decreased ipsilateral forebrain tissue loss. Regional assessment presented in Figure $\mathbf{2 A}$ revealed strong decrease across the different forebrain regions (Mixed Linear Model treating region as a repeated measure, $p=0.001)$. Treatment with curcumin significantly reduced tissue loss in relation to DMSO-treated littermates by $50-90 \%$ in cortex, pyriform cortex, hippocampus, striatum, external capsule, and overall forebrain area $(p<0.05$ in $t$-test). Figure 2B shows large infarct in cortex and hippocampus of the DMSO-treated animal and its sparing in the curcumintreated littermate (Figure 2C).

A similar effect of the curcumin post-treatment was also observed for TUNEL+ cell death (Figures 2D-F). Figure 2D shows that curcumin treatment straight after $60 \mathrm{~min}$ HI significantly reduced the number of TUNEL+ cells compared to DMSO-treated littermates (Mixed Linear Model treating region as a repeated measure $p=0.036$ ), with individual significant decrease of $70-90 \%$ in cortex, pyriform cortex, hippocampus, and overall ( $p<0.05$ in $t$-test). The TUNEL+ cells displayed the typical pyknotic nuclear morphology (Figure 2E-insert, ipsilateral hippocampus DMSO).

In addition to cell death and brain tissue loss, curcumin post-treatment also decreased HI-induced and predominantly ipsilateral reactive astrogliosis and microglial activation. Compared to DMSO-treated animals, their curcumin-treated littermates revealed less GFAP immunoreactivity (Figures 2G,M) with substantially reduced amount of GFAP+ astroglial processes (Figures 2H,I). Assessment across the different forebrain regions through Mixed Linear Model treating region as a repeated measure revealed a clear decrease on the ipsilateral, and milder reduction on the contralateral side (main effect $p=0.005$ and $p=0.0001$, respectively) with individual significant decrease of $30-50 \%$ in all six ipsilateral regions and overall, and of $30 \%$ in contralateral cortex and pyriform cortex $(p<0.05, t$-test).

Curcumin post-treatment had a similar effect on microglia activation score (Figure 2J) based on $\mathrm{aM}$ integrin immunoreactivity (Figures 2K,L). Regional assessment shown in Figure 2J revealed a reduction in activation score in the curcumin-treated group (Mixed Linear Model treating region as a repeated measure, main effect $p=0.019$ ), with significant decrease of $70-90 \%$ in all six individual ipsilateral brain regions $(p<0.05$ in $t$-test).

Compared to DMSO-treated animals, curcumin post-treated littermates had higher levels of myelination assessed through MBP immunoreactivity (Figure 2N), with individual significant increase of $20 \%$ in external capsule $(p<0.05, t$-test).

Curcumin post-treatment also reduced the levels of oxidative stress assessed through iNOS immunoreactivity, compared to DMSO-treated littermates, with individual significant decrease of $50 \%$ in hippocampus (Figure 20, $p<0.05, t$-test).

\section{Immediate Post-treatment With Curcumin Reduces Ipsilateral Volume Loss in Males, Females and Combined (Males + Females), Decreases Myelin Loss in Males, but Does Not Provide Functional Protection Assessed Through the Slipping Test}

To evaluate the long-term effects of curcumin following immediate injection of $200 \mu \mathrm{g} / \mathrm{g}$ post $\mathrm{HI}$, the levels of tissue loss were assessed through Nissl staining, the degree of myelination was evaluated through MBP immunoreactivity, and motor balance and co-ordination were assessed through the slipping test (Rocha-Ferreira et al., 2018) at day 21 post HI (P28).

Intraperitoneal injection of $200 \mu \mathrm{g} / \mathrm{g}$ curcumin straight after $\mathrm{HI}$ decreased tissue loss in males at 21 days post $\mathrm{HI}$ compared to DMSO-treated littermates and untreated $\mathrm{HI}$ controls (Figure 3A). Immediate curcumin treatment had no effect on tissue loss in females compared to DMSO treated or untreated HI controls (Figure 3B). The levels of tissue loss in females were lower compared to males (Figure 3D). Although the differences did not reach significant values, the lower level of tissue loss in females suggests increased susceptibility of males to the insult. The levels of tissue loss in the curcumin group were significantly lower compared to DMSO-treated littermates and untreated HI controls (Figure 3C). Subregional assessment of tissue loss at 21 days post $\mathrm{HI}$ in males (Figure 3E) and combined (males + females) (Figure 3G) suggested that immediate treatment with curcumin at $200 \mu \mathrm{g} / \mathrm{g}$ immediately post HI reduced tissue damage in all studied regions with significant decrease in thalamus. Curcumin treatment did not affect subregional differences in tissue loss in females (Figure 3F). 

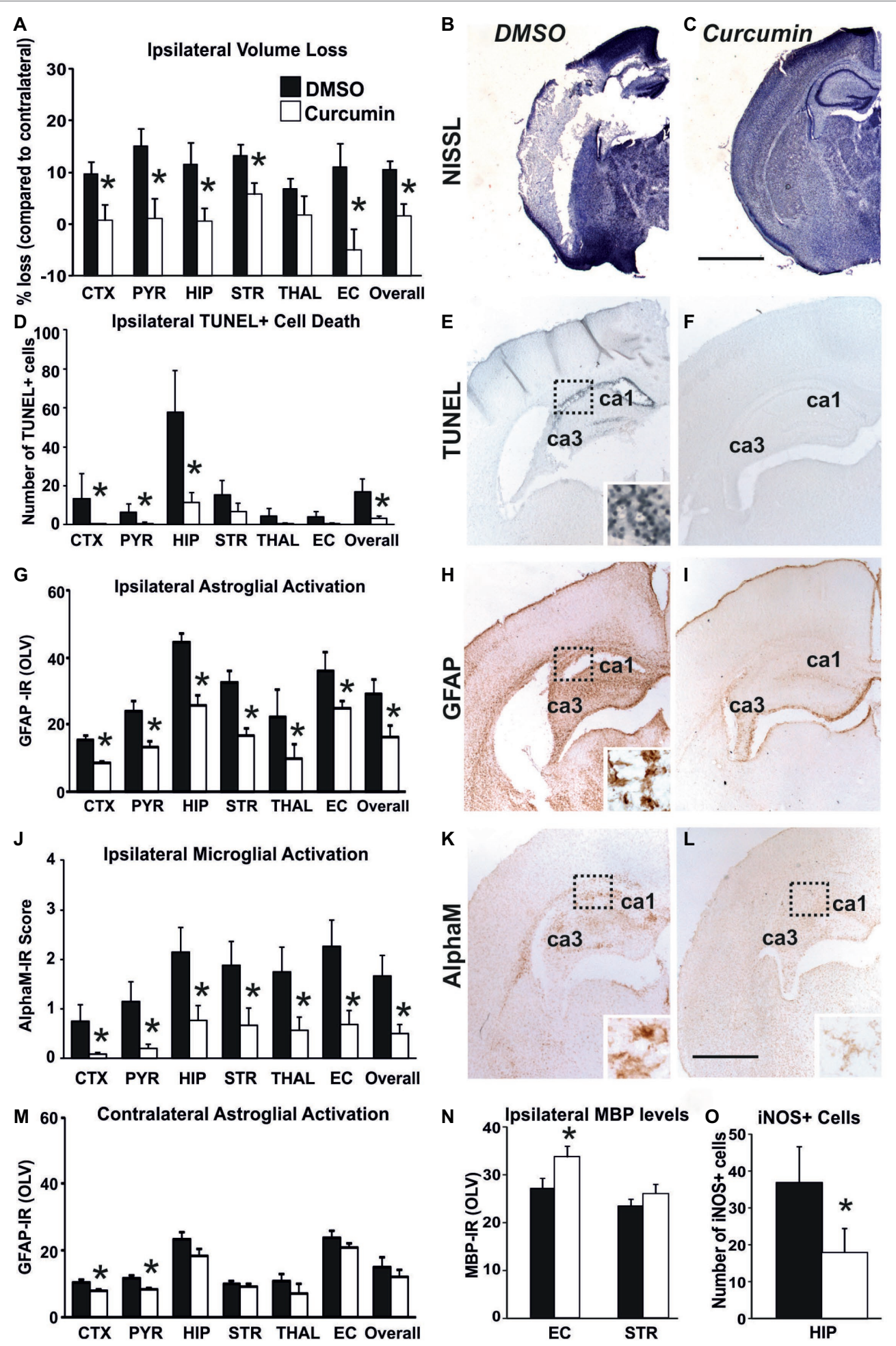

FIGURE 2 | Intraperitoneal injection of curcumin (200 $\mu \mathrm{g} / \mathrm{g} \mathrm{BW})$ in P7 mice immediately post-HI significantly reduces tissue damage, cell death and glial response. (A-C) Ipsilateral forebrain Nissl staining (Cresyl-Violet, at rostral parietal level) - Quantification of ipsilateral brain tissue volume loss (A) at $48 \mathrm{~h}$ following HI-insult of DMSO (B) and curcumin (C) treated animals at $48 \mathrm{~h}$ following HI-insult. Curcumin treatment $(n=11)$ reduced volume loss compared to DMSO-treated littermates $(n=11)$ with significant, individual decrease ( $t$-test) in cortex $(p=0.026)$, pyriform cortex $(p=0.011)$, hippocampus $(p=0.038)$, striatum $(p=0.020)$, external capsule $(p=0.015)$ and overall volume loss $(p=0.003)$. Mixed Linear Model treating region as a repeated measure revealed $p=0.001$. (D-F) TUNEL + staining of dying brain cells with fragmented DNA at $48 \mathrm{~h}$ following HI-insult - Quantification (D) (number of TUNEL+ cells per 20x eye-field, Mean + SEM) and histochemical overview of the ipsilateral forebrain in DMSO (E) and curcumin (F) treated animals. Note the typical pyknotic nuclear morphology of the TUNEL+ cells 


\begin{abstract}
FIGURE 2 | observed in the DMSO group (E- insert, hippocampus) and the lack of such cells in the curcumin group (F). Curcumin treatment reduced TUNEL+ cell death across all 6 examined forebrain regions, with significant, individual decrease ( $t$-test) in cortex $(p=0.04)$, pyriform cortex $(p=0.049)$, hippocampus $(p=0.046)$, and overall $(p=0.048)$. Mixed Linear Model treating region as a repeated measure revealed $p=0.036$. (G-I,M) GFAP immunoreactivity at $48 \mathrm{~h}-\mathrm{Quantification}$ of the ipsilateral (D) and contralateral, non-occluded side (M) in optical luminosity values (OLV, Mean + SEM), and low magnification ipsilateral overview in DMSO (H) and curcumin (I) treated animals. The insert in $\mathbf{H}$ shows higher magnification of the dotted region in rostro-parietal cortex. Note the reduced levels of GFAP immunoreactivity in the curcumin treated animals, with significant, individual decrease (t-test) in ipsilateral cortex $\left(p=4 \times 10^{-5}\right)$, pyriform cortex $(p=0.004)$, hippocampus $(p=0.049)$, striatum $(p=0.001)$, thalamus $(p=0.001)$, external capsule $(p=0.009)$ and overall $(p=0.048)$ in $\mathbf{G}$, and in contralateral cortex $(p=0.01)$ and pyriform cortex $(p=0.0004)$ in $\mathbf{M}$. Mixed Linear Model treating region as a repeated measure revealed $p=0.005$ for the ipsilateral, and $p=0.0001$ for the contralateral side. (J-L) Activation of $\alpha \mathrm{M}+$ microglia - Ipsilateral $\alpha \mathrm{M}$ microglial activation score (J, Mean $+\mathrm{SEM}$ ) and low magnification ipsilateral overview in DMSO (K) and curcumin (L) treated animals. Note the strong microglial activation in DMSO-treated animals with $\alpha \mathrm{M}+$ cells showing phagocytic morphology at high magnification (K-insert, hippocampus), compared to the curcumin treated brains exhibiting a ramified phenotype (L-insert). Curcumin treatment reduced $\alpha M+$ microglial activation across all six examined forebrain regions, with significant, individual decrease ( $t$-test) in cortex $(p=0.03)$, pyriform cortex $(p=0.01)$, hippocampus $(p=0.03)$, striatum $(p=0.04)$, thalamus $(p=0.04)$, external capsule $(p=0.01)$ and overall $(p=0.02)$. Mixed Linear Model treating region as a repeated measure $p=0.019$. DMSO $(n=11)$ and curcumin $(n=11)$ in all assessments. (N) MBP immunoreactivity at $48 \mathrm{~h}$ - Quantification of the ipsilateral external capsule and striatum in optical luminosity values (OLV, Mean + SEM). Note the increased levels of MBP immunoreactivity in the curcumin treated animals, with significant, individual increase ( $t$-test) in ipsilateral external capsule $(p=0.045)$. Mixed Linear Model treating region as a repeated measure $p=0.056$. (0) iNOS immunoreactivity at $48 \mathrm{~h}$ - Quantification of the number of iNOS+ cells in ipsilateral hippocampus (number of iNOS+ cells per 20x eye-field, Mean + SEM). Note the reduced number of iNOS+ cells ( $t$-test) in the curcumin treated group compared to DMSO-treated littermates $(p=0.041)$. $\left({ }^{*} p<0.05\right)$. Abbreviations: CTX, cerebral cortex; EC, external capsule; HIP, hippocampus; PYR, pyriform cortex; STR, striatum; THAL, thalamus. Scale bars: $\mathbf{( B , C )}=2,000 \mu \mathrm{m} ;(\mathbf{E}, \mathbf{F}, \mathbf{H}, \mathbf{I}, \mathbf{K}, \mathbf{L})=1,000 \mu \mathrm{m}$; inserts $=62 \mu \mathrm{m}$.
\end{abstract}

Compared to untreated male HI control animals, curcumin post-treated littermates had an increase in myelination assessed through MBP immunoreactivity (Figure $3 \mathbf{H}$ ) of $23 \%$ in striatum $(p<0.05$, two-way ANOVA). Immediate post-HI treatment with $200 \mu \mathrm{g} / \mathrm{g}$ curcumin did not affect myelin loss in females (Figure 3I) or combined (males + females) (Figure 3J).

Assessment of motor balance and co-ordination through slipping test at 21 days post $\mathrm{HI}$ of animals treated immediately after the insult with $200 \mu \mathrm{g} / \mathrm{g}$ curcumin, DMSO, or HI controls showed slightly decreased number of missed steps in curcumintreated males (Figure 3K), females (Figure 3L) and combined (males + females) (Figure 3M); however, the differences did not reach significance (Kruskal-Wallis test).

\section{Immediate Post-treatment With Curcumin Reduces Brain Damage Following Neonatal HI-Insult in a Dose-Dependent Manner}

Curcumin dose of $200 \mu \mathrm{g} / \mathrm{g}$ BW resulted in significant neuroprotection (Figure 2). To determine whether a lower dose of curcumin post-treatment immediately after the HI-insult would provide neuroprotective effects, three groups of animals were injected intraperitoneally with doses of 50,22, and $10 \mu \mathrm{g} / \mathrm{g}$ BW curcumin in $0.5 \mu \mathrm{l} / \mathrm{g}$ BW DMSO. The control littermates in each group received the same volume of DMSO only. Brain damage markers (tissue loss, TUNEL+ cell death, reactive astrogliosis, and microglial activation) were assessed at $48 \mathrm{~h}$ post-HI. As shown in Figure 4, the dose of $50 \mu \mathrm{g} / \mathrm{g}$ (Figure 4A) significantly reduced the levels of microglial activation (Mixed Linear Model treating region as a repeated measure $p=0.035$ ), with significant individual decrease of $50-65 \%$ in cortex, hippocampus, striatum, and external capsule ( $p<0.05, t$-test). Similarly, the dose of $50 \mu \mathrm{g} / \mathrm{g}$ BW curcumin significantly reduced the levels of ipsilateral tissue loss (Mixed Linear Model treating region as a repeated measure $p=0.0001$ ), with significant individual decrease of $75-90 \%$ in cortex, hippocampus, and overall (Supplementary Figure S1A, $p<0.05, t$-test). The levels of TUNEL+ cell death were also reduced by the dose of $50 \mu \mathrm{g} / \mathrm{g}$ BW curcumin (Mixed Linear Model treating region as a repeated measure $p=0.016$ ) with significant individual reduction of 75-90\% in hippocampus and external capsule (Supplementary Figure S1D, $p<0.05, t$-test). The dose of $50 \mu \mathrm{g} / \mathrm{g}$ markedly reduced also the levels of ipsilateral reactive astrogliosis (Mixed Linear Model treating region as a repeated measure $p=0.014$ ), with significant individual reduction of $44-55 \%$ in pyriform cortex, hippocampus, thalamus, and external capsule (Supplementary Figure S1G, $p<0.05, t$-test). In a similar pattern, the dose of $50 \mu \mathrm{g} / \mathrm{g}$ significantly reduced contralateral astroglial activation (Mixed Linear Model treating region as a repeated measure $p=0.005)$, with significant individual decrease in pyriform cortex $(p<0.05$, Supplementary Figure S1J). The doses of 22 and $10 \mu \mathrm{g} / \mathrm{g}$ BW curcumin did not have an effect on microglial activation (Figures 4B,C), ipsilateral volume loss, TUNEL+ cell death or reactive astrogliosis (Supplementary Figures S1B,C,E,F,H,I,K,L).

\section{Delayed Post-treatment With Curcumin at 60 and 120 min Post HI Reduces Brain Damage Following Neonatal HI-Insult}

To investigate whether delayed application of curcumin would provide similar neuroprotection to the one achieved through immediate post-HI treatment, $200 \mu \mathrm{g} / \mathrm{g}$ BW curcumin was applied at 60 and 120 min following a neonatal $\mathrm{HI}$ insult and the brain damage markers were assessed at $48 \mathrm{~h}$ post $\mathrm{HI}$. Treatment at 60 min reduced volume loss by $60-70 \%$ in cortex, hippocampus, striatum, thalamus, and overall compared to $\mathrm{HI}$ controls (Figure 5A, $p<0.05$, Kruskal-Wallis test with Bonferroni correction), but had no effect compared to DMSO-treated littermates and no main effect of the treatment was observed. Application of curcumin at $60 \mathrm{~min}$ post HI significantly reduced TUNEL+ cell death compared to HI- and DMSO-treated littermates (Figure 5C, $p<0.05$, Kruskal-Wallis test). Pairwise comparison between the curcumin treated and $\mathrm{HI}$ groups revealed significant $80-90 \%$ decrease of the number of TUNEL+ cells in cortex, pyriform cortex, hippocampus and overall (Figure 5C, $p<0.05$, Kruskal-Wallis test with Bonferroni correction). Compared to DMSO-treated littermates curcumin 


\section{Ipsilateral Volume Loss at P28 (d21)}
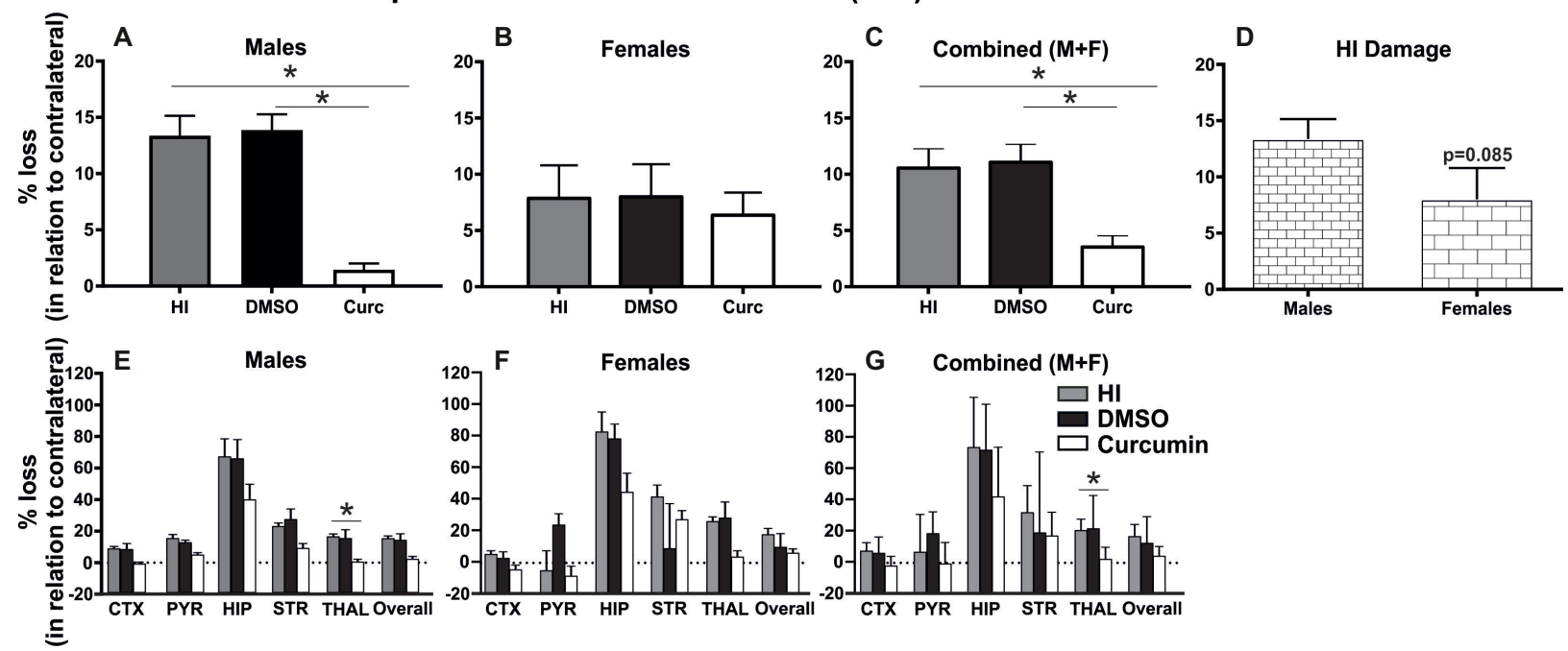

Ipsilateral MBP levels
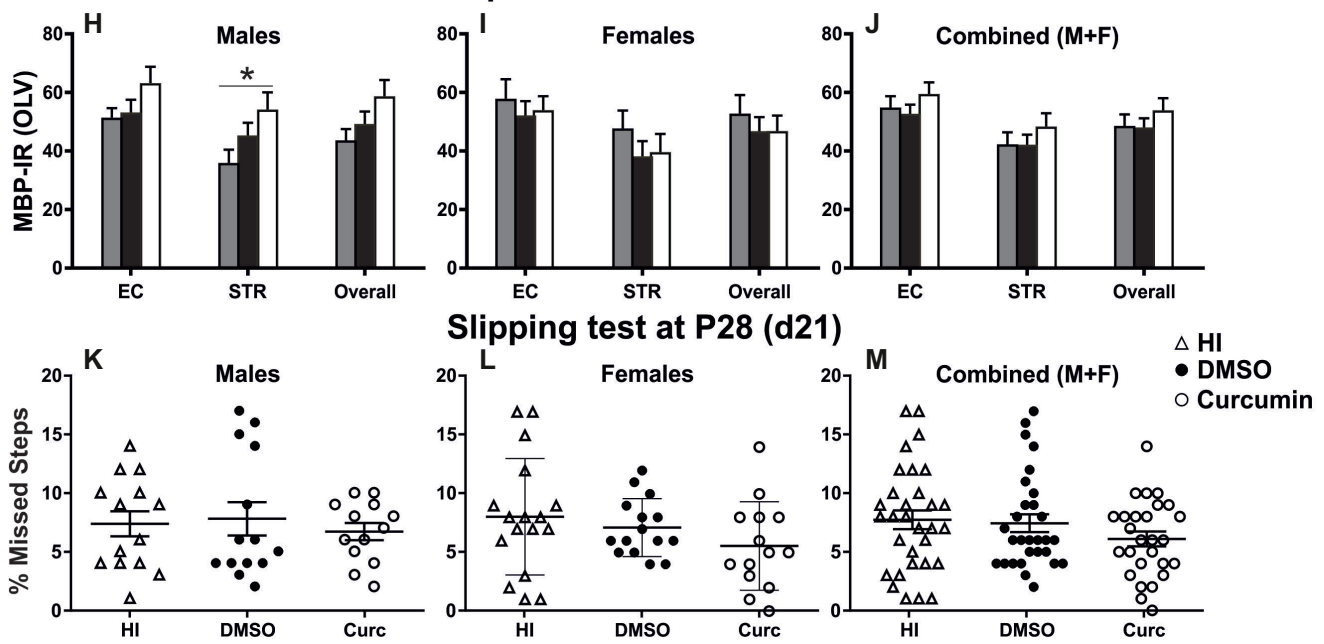

FIGURE 3 | Intraperitoneal injection of curcumin in P7 mice post-HI reduces ipsilateral volume loss in males, females and combined (males + females), decreases myelin loss in males, but does not provide functional protection assessed through slipping test. (A) Intraperitoneal injection of 200 $\mu \mathrm{g} / \mathrm{g}$ curcumin straight after HI decreased tissue loss at 21 days post $\mathrm{HI}(\mathrm{P} 28)$ in males compared to DMSO-treated littermates and untreated $\mathrm{HI}$ controls $(\mathrm{curcumin} n=11$, DMSO $n=8, \mathrm{HI} n=9$; curcumin vs. HI $p=0.0017$, curcumin vs. DMSO $p=0.0003$ ). (B) Curcumin treatment at $200 \mu \mathrm{g} / \mathrm{g}$ straight after $\mathrm{HI}$ did not affect tissue loss at 21 days post HI in females compared to DMSO-treated littermates or to untreated HI controls. (curcumin $n=8$, DMSO $n=7$, HI $n=8$ ). (C) The levels of tissue loss in the curcumin treated animals decreased compared to DMSO-treated littermates and untreated $\mathrm{HI}$ controls (curcumin $n=19$, DMSO $n=15, \mathrm{HI} n=17$; curcumin vs. HI $p=0.0024$, curcumin vs. DMSO $p=0.0014)$. (D) The level of tissue loss observed in females was lower compared to the males, however the differences did not reach significant values $(p=0.085)$. This suggests higher susceptibility of the males to $H \mathrm{H}$ insult. (E-G) Subregional assessment of tissue loss at 21 days post $\mathrm{HI}$ in males (E, curcumin $n=11$, DMSO $n=8, \mathrm{HI} n=9$ ), females (F, curcumin $n=8$, DMSO $n=7, \mathrm{HI} n=8)$ and combined (males + females) (G, curcumin $n=19$, DMSO $n=15, \mathrm{HI} n=17)$. Curcumin treatment with $200 \mu \mathrm{g} / \mathrm{g}$ immediately post $\mathrm{HI}$ resulted in a reduction of subregional tissue loss in all studied regions in males (E) and combined (males + females) (G) with significant differences observed in thalamus (males $p=0.0292$, combined (males + females) $p=0.0242$ ). (F) Curcumin treatment had no effect on subregional differences in tissue loss in females. (H-J) MBP immunoreactivity at 21 days post $\mathrm{HI}(\mathrm{P} 28)$. Quantification of the ipsilateral external capsule, striatum, and overall in optical luminosity values (OLV, Mean + SEM). (H) In males curcumin treatment with 200 $\mu g / g$ immediately post HI resulted in increased levels of MBP immunoreactivity compared to untreated $\mathrm{HI}$ controls (curcumin $n=11$, DMSO $n=8, \mathrm{HI} n=9$ ), with significant differences in striatum (Two-way ANOVA with post hoc Tukey's test, $p=0.042)$. In females (I, curcumin $n=8$, DMSO $n=7, \mathrm{HI} n=8)$ and combined (males + females) $(\mathbf{J}$, curcumin $n=19$, DMSO $n=15, \mathrm{HI} n=17$ ), immediate treatment with $200 \mu \mathrm{g} / \mathrm{g}$ curcumin had no effect on MBP immunoreactivity. (K-M) Curcumin treatment with $200 \mu \mathrm{g} / \mathrm{g}$ immediately post $\mathrm{HI}$ did not affect the number of missed steps (slipping test) at 21 days (P28) post HI in males (K, curcumin $n=13$, DMSO $n=14$, HI $n=14$ ), females (L, curcumin $n=14$, DMSO $n=15, \mathrm{HI} n=17$ ) and combined (males + females) (M, curcumin $n=27$, DMSO $n=29, \mathrm{HI} n=31)$. ( $\left.{ }^{*} p<0.05\right)$. Abbreviations: CTX, cerebral cortex; EC - external capsule; HIP - hippocampus; PYR, pyriform cortex; STR, striatum; THAL, thalamus.

treatment at 60 min post $\mathrm{HI}$ significantly reduced TUNEL+ cell death by $80-90 \%$ in cortex, pyriform cortex, hippocampus and overall (Figure 5C, $p<0.05$, Kruskal-Wallis test with
Bonferroni correction). Administration of curcumin at $60 \mathrm{~min}$ post $\mathrm{HI}$ reduced microglial activation compared to DMSOtreated littermates and $\mathrm{HI}$ controls, although the main effect 


\section{Ipsilateral Microglial Activation}

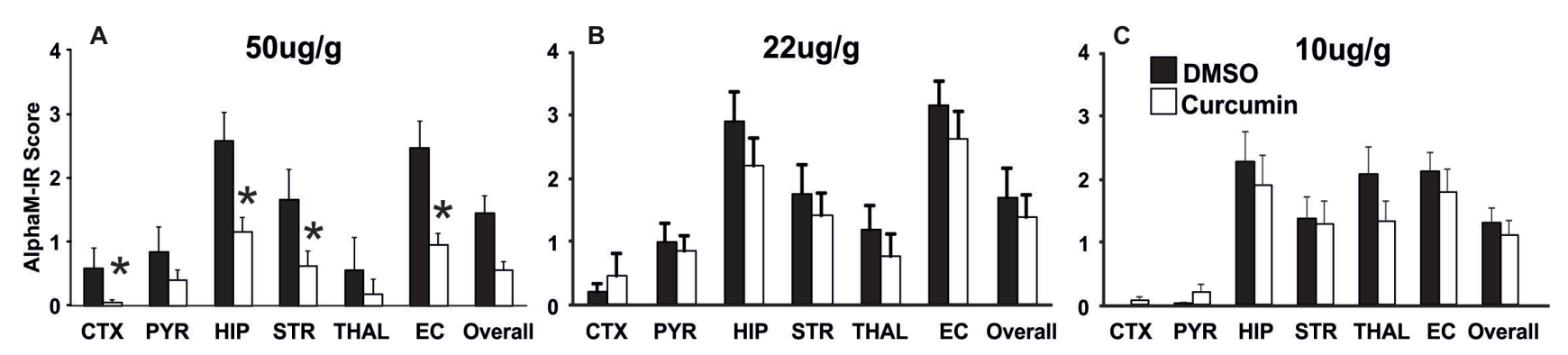

FIGURE 4 | Intraperitoneal injection of curcumin in P7 mice post-HI significantly reduces ipsilateral $\alpha \mathrm{M}$ microglial activation score in a dose dependent manner. (A) Intraperitoneal injection of $50 \mathrm{\mu g} / \mathrm{g}$ BW curcumin decreased $\alpha \mathrm{M}+$ microglial activation compared to DMSO-treated littermates, with significant, individual decrease ( $t$-test) in cortex $(p=0.038)$, hippocampus $(p=0.035)$, striatum $(p=0.035)$ and external capsule $(p=0.046)$. Mixed Linear Model treating region as a repeated measure revealed $p=0.035$. (B,C) Intraperitoneal curcumin injection of $22 \mu \mathrm{g} / \mathrm{g}(\mathbf{B})$ or $10 \mu \mathrm{g} / \mathrm{g}(\mathbf{C})$ did not have an effect on $\alpha \mathrm{M}+$ microglial activation compared to DMSO-treated littermates. (A, curcumin $50 \mu \mathrm{g} / \mathrm{g} n=8$, DMSO $n=6$, B, curcumin $22 \mu \mathrm{g} / \mathrm{g} n=12, \mathrm{DMSO} n=10, \mathbf{C}, \mathrm{curcumin} 10 \mu \mathrm{g} / \mathrm{g} n=11, \mathrm{DMSO}$ $n=11) .\left({ }^{*} p<0.05\right)$ Abbreviations: CTX, cerebral cortex; EC, external capsule; HIP, hippocampus; PYR, pyriform cortex; STR, striatum; THAL, thalamus.

of treatment did not reach significance (Figure 5E). Curcumin treatment at $60 \mathrm{~min}$ post $\mathrm{HI}$ reduced microglial activation compared to HI controls with significant, individual decrease of $30 \%$ in external capsule (Figure 5E, $p<0.05$, Kruskal-Wallis test with Bonferroni correction). Compared to DMSO-treated littermates curcumin treatment at $60 \mathrm{~min}$ post-HI reduced microglial activation with significant, individual decrease of $90 \%$ in cortex (Figure 5E, $p<0.05$, Kruskal-Wallis test with Bonferroni correction). Curcumin application at $60 \mathrm{~min}$ post HI did not affect ipsilateral astroglial activation (Figure 5G).

Application of curcumin at 120 min post HI surprisingly increased the level of tissue loss but there was no significant main effect of treatment ( $p>0.05$, one-way ANOVA). However pairwise comparison revealed significant increase of $50 \%$ in volume loss in the cortex of curcumin treated compared to $\mathrm{HI}$ and to DMSO-treated littermates (Figure 5B, $p<0.05$, one-way ANOVA with Bonferroni correction). Delayed administration of curcumin at 120 min post HI significantly reduced the number of TUNEL+ cells compared to HI- and DMSO-treated littermates (Figure 5D, $p<0.05$, one-way ANOVA). Pairwise comparison revealed significant 60-90\% decrease of TUNEL+ cell death in curcumin treated compared to $\mathrm{HI}$ animals in hippocampus, striatum and overall (Figure 5D, $p<0.05$, one-way ANOVA with Bonferroni correction). Pairwise comparison between curcumin and DMSO-treated animals revealed significant $50-85 \%$ decrease of TUNEL+ cell death in hippocampus, striatum and overall (Figure 5D, $p<0.05$, one-way ANOVA with Bonferroni correction). Administration of curcumin at 120 min post $\mathrm{HI}$ reduced microglial activation compared to $\mathrm{HI}$ - and DMSO-treated littermates (Figure 5F, $p<0.05$, Kruskal-Wallis test). Pairwise comparison between curcumin treated and HI animals revealed significant $40-60 \%$ decrease of microglial activation in pyriform cortex, hippocampus, striatum, thalamus, external capsule and overall (Figure 5F, $p<0.05$, Kruskal-Wallis test with Bonferroni correction). Pairwise comparison revealed significant 50\% decrease of microglial activation in curcumin compared to DMSO-treated littermates in pyriform cortex, external capsule and overall (Figure 5F, $p<0.05$, Kruskal-Wallis test with Bonferroni correction). Administration of curcumin at $120 \mathrm{~min}$ post $\mathrm{HI}$ reduced ipsilateral reactive astrogliosis in cortex by $18 \%$ compared to $\mathrm{HI}$ and by $38 \%$ compared to DMSO-treated littermates (Figure $\mathbf{5 H}$, $p<0.05$, Kruskal-Wallis test with Bonferroni correction); however, no main effect of the treatment was observed.

\section{Post-treatment With Curcumin Immediately Post HI Has No Effect on the Levels of Cellular Proliferation at $48 \mathrm{~h}$}

To determine whether the neuroprotective effects of immediate curcumin treatment post $\mathrm{HI}$ are due to a boost of cellular proliferation, we assessed the number of $\mathrm{BrdU}$ positive cells in P7 naïve animals, naïve animals injected with $200 \mu \mathrm{g} / \mathrm{g}$ curcumin, and HI animals injected immediately post insult with either $200 \mu \mathrm{g} / \mathrm{g}$ curcumin or DMSO. The number of BrdU+ cells in naïve untreated animals or naïve and $\mathrm{HI}$ animals treated with curcumin or DMSO did not differ between the groups in any of the tested brain regions (Figure 6A). Although curcumin treatment did not result in differences in cell proliferation levels in naïve or HI animals, we assessed its effect on the proliferation of different cell types, i.e., microglia, astroglia, oligodendrocyte precursors and neurons through double labeling for BrdU and IBA1 (microglia), GFAP (astroglia), NG2 (oligodendrocyte precursors), and NeuN (neurons) and calculation of the percentage of the double positive cells over the BrdU positive ones.

Curcumin treatment of naïve or HI animals immediately after insult did not affect the percentage of IBA1 and BrdU double positive (Figure 6B), GFAP and BrdU double positive (Figure 6C), NG2 and BrdU double positive (Figure 6D), or NeuN and BrdU double positive (Figure 6E) over BrdU positive cells per region and group. The percentage of proliferating microglia (Figures 6F,N,J), astroglia (Figures 6G, $\mathrm{O}, \mathrm{K}$ ), and oligodendrocytes (Figures $6 \mathbf{H}, \mathbf{P}, \mathrm{L})$ was relatively low $(10-20 \%$ of the total number of BrdU positive cells) compared to the neurons which comprised $80-90 \%$ of the BrdU positive cells (Figures 6I,Q,M). 


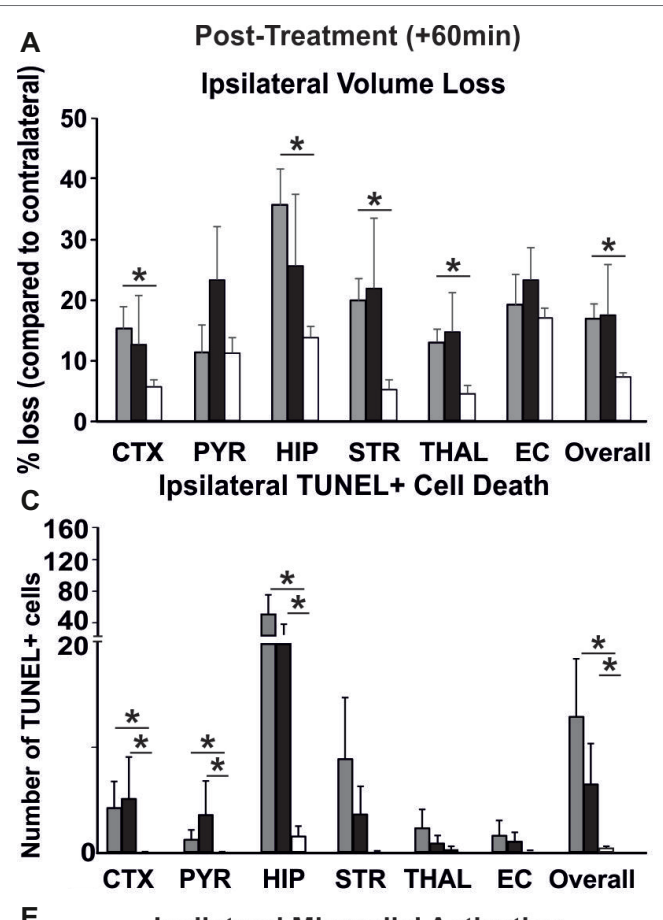

\section{B Post-Treatment (+120min) \\ Ipsilateral Volume Loss}
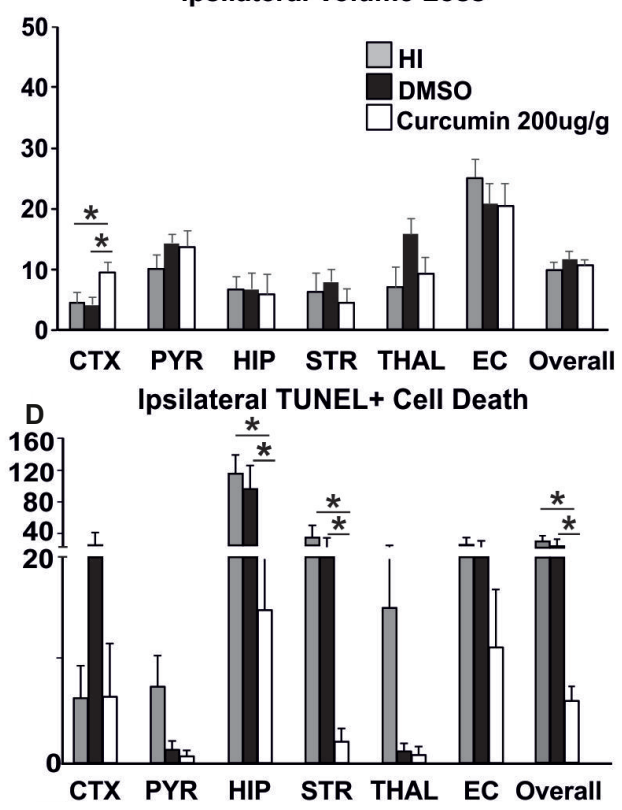

F Ipsilateral Microglial Activation
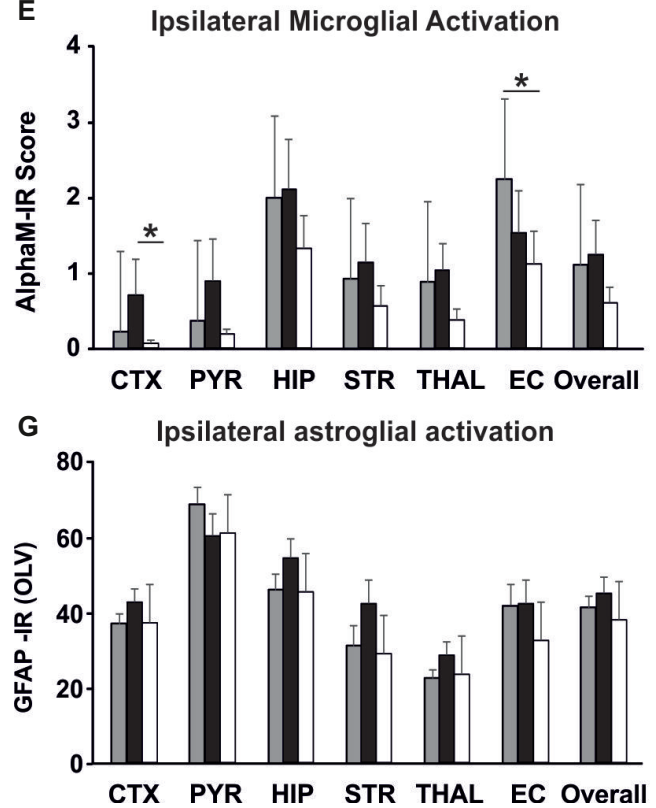
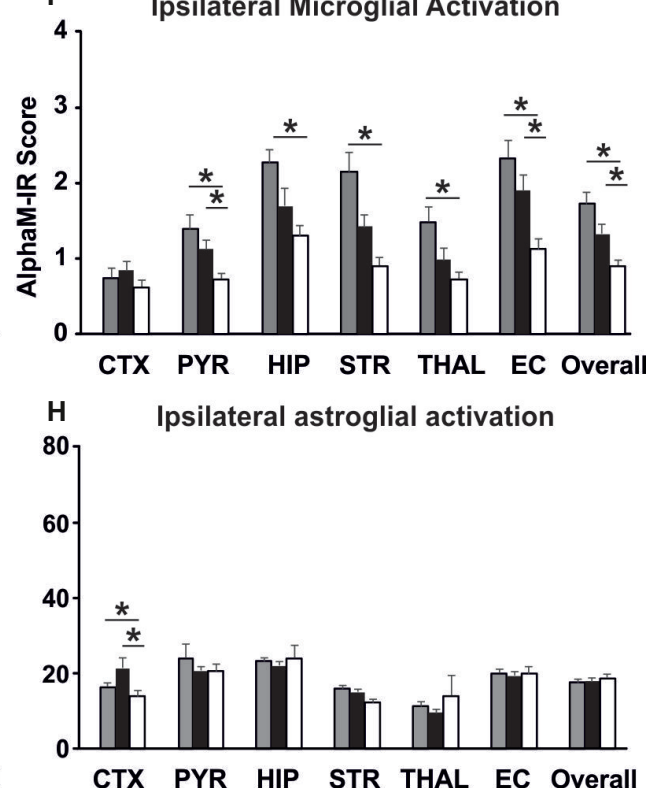

FIGURE 5 | Intraperitoneal injection of curcumin (200 $\mathrm{\mu g} / \mathrm{g} \mathrm{BW})$ in P7 mice at 60 min or 120 min post-HI significantly reduces tissue damage, cell death and glial response. (A) Curcumin treatment at 60 min post $\mathrm{HI}(n=8)$ reduced volume loss compared to HI controls $(n=10)$ with significant, individual decrease (KruskalWallis test with Bonferroni correction) in cortex ( $p=0.036)$, hippocampus $(p=0.007)$, striatum $(p=0.003)$, thalamus ( $p=0.005)$, and overall volume loss $(p=0.002)$. However, no main effect of the treatment was observed. No differences were registered between curcumin and DMSO $(n=7)$ treated littermates. (B) Administration of curcumin at 120 min post $\mathrm{HI}(n=7)$ surprisingly increased the level of tissue loss however no significant main effect of treatment was observed. Pairwise comparison (one-way ANOVA with Bonferroni correction) revealed significant increase of volume loss in cortex in curcumin treated compared to $\mathrm{HI}(n=7$, $p=0.05)$ and to DMSO-treated littermates $(n=7, p=0.017)$. (C) Curcumin treatment at 60 min post HI reduced TUNEL+ cell death compared to HI- and DMSOtreated littermates (Kruskal-Wallis test). Pairwise comparison (Bonferroni correction) revealed significant decrease of TUNEL+ cell death in curcumin treated compared to $\mathrm{HI}$ animals in cortex $(p=0.064)$, pyriform cortex $(p=0.064)$, hippocampus $(p=0.003)$ and overall $(p=0.001)$. Pairwise comparison (Bonferroni correction) revealed significant decrease of TUNEL+ cell death in curcumin compared to DMSO-treated animals in cortex $(p=0.003)$, pyriform cortex ( $p=0.013)$, hippocampus ( $p=0.042)$, and overall $(p=0.012)$. (D) Administration of curcumin at 120 min post HI reduced TUNEL+ cell death compared to HI- and DMSOtreated littermates (tone-way ANOVA, main effect $p=0.001$ ). Pairwise comparison (Bonferroni correction) revealed significant decrease of TUNEL+ cell death in curcumin treated compared to $\mathrm{HI}$ animals in hippocampus $(p=0.002)$, striatum $(p=0.02)$, and overall $(p=0.001)$. Pairwise comparison (Bonferroni correction) revealed significant decrease of TUNEL+ cell death in curcumin compared to DMSO-treated littermates in hippocampus $(p=0.012)$, striatum $(p=0.022)$ and overall $(p=0.002)$. (E) Administration of curcumin at 60 min post $\mathrm{HI}$ reduced microglial activation compared to DMSO-treated littermates and $\mathrm{HI}$ controls, 
FIGURE 5 | although the main effect of treatment did not reach significance. However, compared to HI controls, curcumin treatment reduced microglial activation with significant, individual decrease (Kruskal-Wallis test with Bonferroni correction) in external capsule ( $p=0.042$ ). Compared to DMSO-treated littermates curcumin treatment at 60 min post-HI reduced microglial activation with significant, individual decrease (Kruskal-Wallis test with Bonferroni correction) in cortex ( $p=0.028)$. (F) Administration of curcumin at 120 min post $\mathrm{HI}$ reduced microglial activation compared to $\mathrm{HI}$ - and DMSO-treated littermates (Kruskal-Wallis test, main effect $p=0.002)$. Pairwise comparison (Bonferroni correction) revealed significant decrease of microglia activation in curcumin treated compared to HI animals in pyriform cortex $(p=0.03)$, hippocampus $(p=0.002)$, striatum $(p=0.002)$, thalamus $(p=0.01)$, external capsule $(p=0.006)$ and overall $(p=0.004)$. Pairwise comparison (Bonferroni correction) revealed significant decrease of microglia activation in curcumin compared to DMSO-treated littermates in pyriform cortex ( $p=0.018$ ), external capsule ( $p=0.022)$, and overall $(p=0.016)$. (G) Curcumin treatment at 60 min post HI did not affect ipsilateral reactive astrogliosis. (H) Administration of curcumin at 120 min post $\mathrm{HI}$ reduced ipsilateral reactive astrogliosis (Kruskal-Wallis test with Bonferroni correction) in cortex compared to $\mathrm{HI}(p=0.049)$ and DMSOtreated littermates $(p=0.006)$, however no main effect of the treatment was observed. $\left({ }^{*} p<0.05\right)$. Abbreviations: CTX, cerebral cortex; EC - external capsule; HIP, hippocampus; PYR, pyriform cortex; STR, striatum; THAL, thalamus.
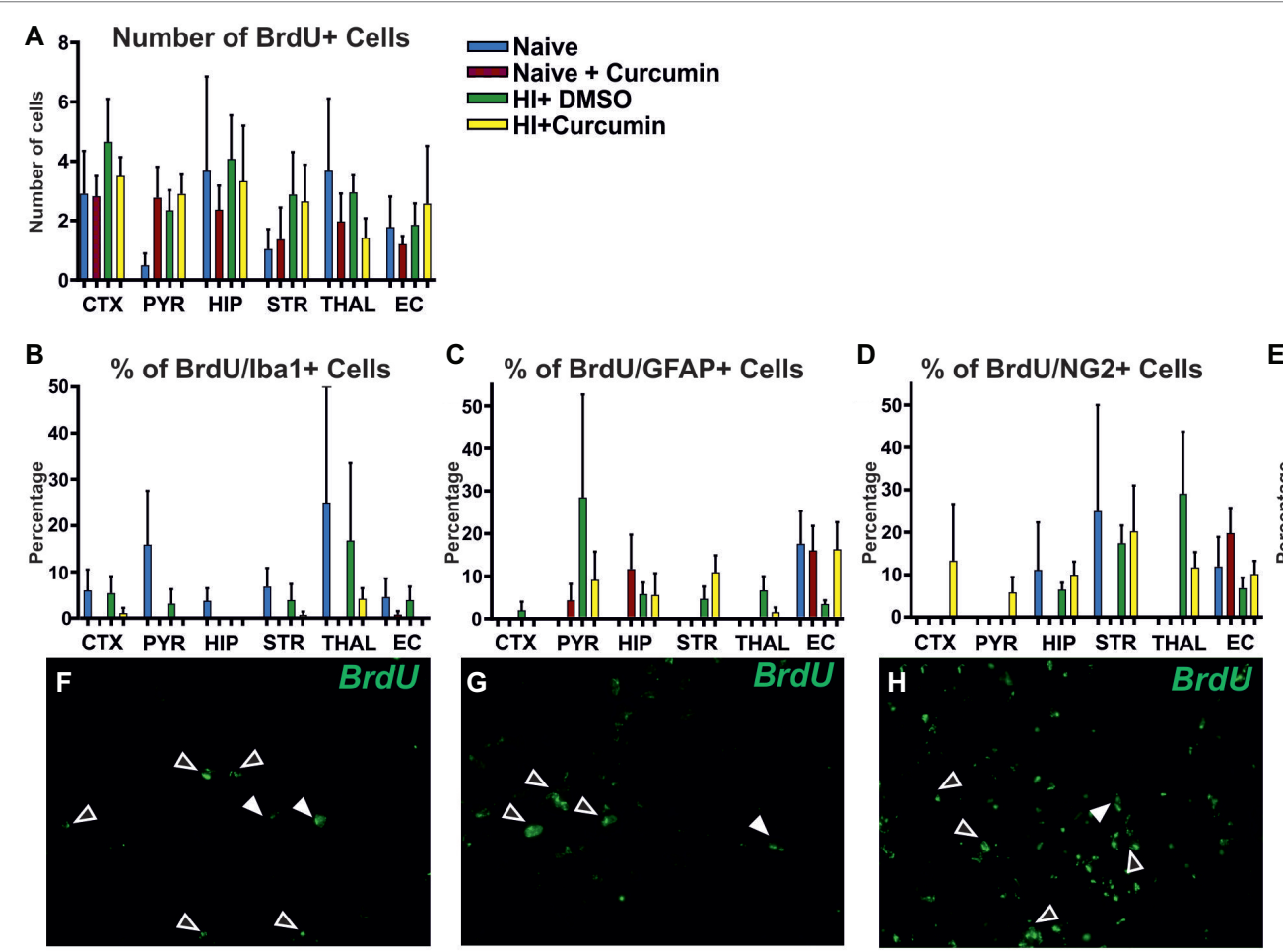

$\%$ of BrdU/NeuN+ Cells
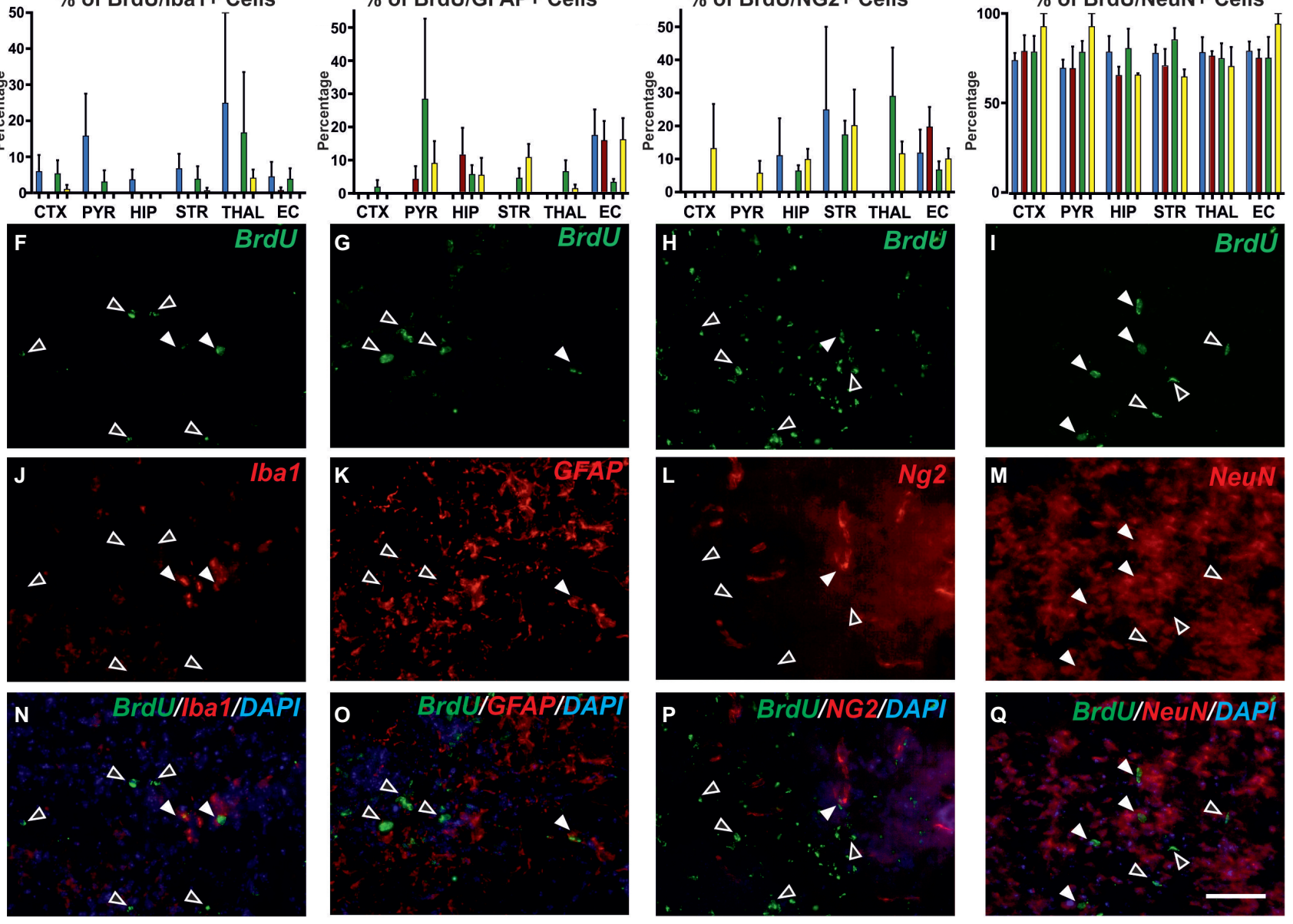

FIGURE 6 | Intraperitoneal injection of curcumin $(200 \mu \mathrm{g} / \mathrm{g}$ BW) in P7 mice immediately post-HI has no effect on the levels of proliferation at $48 \mathrm{~h}$.

(A) Assessment of the number of BrdU+ cells in P7 naïve untreated animals or naïve and $\mathrm{HI}$ animals treated with curcumin or DMSO did not show differences between the groups in any of the regions. (naïve $n=4$, naïve + curcumin $n=5, \mathrm{HI}+\mathrm{DMSO} n=7, \mathrm{HI}+$ curcumin $n=8$ ). (B) Curcumin treatment in naïve or $\mathrm{HI}$ animals immediately after insult did not affect the percentage of IBA1 and BrdU double positive over BrdU+ cells per region and group. The percentage of proliferating IBA1+ cells was low (10-20\% of the BrdU+ cells). (C) The percentage of GFAP and BrdU double positive over BrdU+ cells per region and (Continued) 


\begin{abstract}
FIGURE 6 I group was not affected by curcumin treatment in naïve or HI animals. The percentage of proliferating GFAP+ cells was low (10-20\% of the BrdU+ cells). (D) The percentage of NG2 and BrdU double positive over BrdU+ cells per region and group was not affected by curcumin treatment in naïve or HI animals. The percentage of proliferating NG2+ cells was low (10-20\% of the BrdU+ cells). (E) The percentage of NeuN and BrdU double positive over BrdU+ cells per region and group was not affected by curcumin treatment in naïve or $\mathrm{HI}$ animals. The percentage of proliferating NeuN+ cells comprised $80-90 \%$ of the BrdU+ cells and was the highest of all four studied cell types. (F,J,N) Immunofluorescence for rat polyclonal anti-BrdU (F,N) in green superimposed on the rabbit polyclonal anti-IBA1 $\mathbf{( J , N )}$ in red, and nuclear DAPI fluorescence in blue (N). Note the co-localization of BrdU and IBA1 (white arrows) and the lack of such co- localization (empty arrows). (G,K,O) Immunofluorescence for rat polyclonal anti-BrdU (G,O) in green superimposed on the rabbit polyclonal anti-GFAP (K,O) in red, and nuclear DAPI fluorescence in blue (0). Note the co-localization of BrdU and GFAP (full arrows) and the lack of such co-localization (empty arrows). (H,L,P) Immunofluorescence for rat polyclonal anti-BrdU (H,P) in green superimposed on the guinea pig polyclonal anti-NG2 (L,P) in red, and nuclear DAPI fluorescence in blue (P). Note the co-localization of BrdU and NG2 (full arrows) and the lack of such co-localization (empty arrows). (I,M,Q) Immunofluorescence for rat polyclonal anti-BrdU (I,Q) in green superimposed on the mouse monoclonal anti-NeuN (M,Q) in red, and nuclear DAPI fluorescence in blue (Q). Note the high level of co-localization of BrdU and NeuN (full arrows) and the lack of such co-localization (empty arrows). Abbreviations: CTX, cerebral cortex; EC - external capsule; HIP - hippocampus; PYR, pyriform cortex; STR, striatum; THAL, thalamus. Scale bar: $126 \mu \mathrm{m}$.
\end{abstract}

\section{Post-treatment With Curcumin Immediately Post-HI Decreases Phosphorylated STAT3 in Hippocampus, While Increasing Ipsilateral PHB Protein Levels at $1 \mathrm{~h}$}

Western Blot analyses for phosphorylated STAT3 Y705 (pSTAT3 Y705) in ipsilateral and contralateral hippocampus from animals with no treatment (HI), DMSO- or $200 \mu \mathrm{g} / \mathrm{g}$ curcumin treatment and $1 \mathrm{~h}$ recovery demonstrated a slight bilateral increase in pSTAT3 Y705 in the DMSO-treated group compared to the HI controls; however, no significant differences were achieved (Figure 7A). Curcumin treatment significantly decreased pSTAT3 Y705 on the contralateral side compared to DMSO-treated animals but did not show significant reduction compared to the HI group. Curcumin treatment did not result in significant changes in the ipsilateral side (Figure 7A). Western Blot analyses for phosphorylated STAT3 S727 (pSTAT3 S727) under the same conditions showed a slight ipsilateral increase of pSTAT3 S727 in the DMSO-treated compared to HI control animals; however, significant differences were not reached (Figure 7B). Curcumin treatment significantly reduced the levels of pSTAT3 S727 compared to the DMSO-treated animals, but had no effect in comparison to the HI littermate controls. No differences between the three groups were registered on the contralateral side (Figure 7B).

Western Blots analyses for PHB at $1 \mathrm{~h}$ post-HI of animals with $\mathrm{HI}, \mathrm{DMSO}$, or $200 \mu \mathrm{g} / \mathrm{g}$ curcumin treatment showed ipsilateral increase of $\mathrm{PHB}$ protein levels in the curcumin compared to DMSO-treated animals, but not to HI littermate controls (Figure 7C). No significant differences were observed between the DMSO-treated animals and the HI littermate controls. No differences between the three groups were registered on the contralateral side (Figure 7C).

The values represent relative densitometry compared to $\beta$-actin, which was used as the internal loading control.

\section{DISCUSSION}

As shown in the current study, in a Rice-Vannucci model of severe HI insult in P7 mice, immediate, as well as delayed (60 or $120 \mathrm{~min}$ ) application of curcumin after the HI insult clearly reduced forebrain cell death and tissue loss, as well as microglial and astroglial activation, in a dose dependent manner at $48 \mathrm{~h}$ post HI (Figures 2,4,5; Supplementary Figure S1). Our results show higher levels of MBP in the external capsule of the curcumin treated group at $48 \mathrm{~h}$ post $\mathrm{HI}$ and in the striatum of males at 21 days post $\mathrm{HI}$, suggesting protected myelination compared to the DMSO-treated littermates and untreated HI control littermates (Figures 2N,3H). These data were in line with other studies demonstrating that decrease in MBP loss is associated with neuroprotection of white matter following neonatal HI injury (Carlsson et al., 2012; Cui et al., 2017). Our data were also in line with the results from other groups registering maintenance of myelin structure and upregulation of MBP expression in the cerebellar white matter in a model of sodium arsenite induced neurotoxicity in developing rat cerebellum (Kaushal et al., 2014). Surprisingly, we did not register similar effect in females (Figure 3I), which was also reflected in the combined (males + females) assessment (Figure 3J). This is in line with the fact that male sex is a well-established risk factor for poor neurodevelopmental outcome after birth asphyxia and that the male hippocampus, normally larger than the female, undergoes a greater volume loss compared to females (Mayoral et al., 2009). We also observed decrease in the number of iNOS positive cells in hippocampus following curcumin treatment post-HI (Figure 2O) suggesting reduction in oxidative stress. Our data is in line with previous studies in a rat model of neonatal HI where curcumin treatment prevented myelin loss, reduced iNOS expression and decreased caspase-3 dependent apoptosis (Cui et al., 2017). Additionally, our results also show reduction in TUNEL+ cell death and tissue loss (Figure 2, Supplementary Figure S1) thus broadening the spectrum of assessed cell death.

Our experiments showed attenuation of tissue loss at 21 days post HI in males, females and combined (males + females), (Figures 3A-C), however the effects were more pronounced in the male group with subregional significant differences observed in thalamus of males and combined (males + females) (Figures 3E,G). The levels of damage in the female group were lower compared to the males (Figure 3D). Sex-related susceptibility to brain damage is probably the reason for no visible effect of the curcumin treatment in females (Figures 3B,F). Immediate post-HI treatment with curcumin slightly improved motor balance and co-ordination, assessed through the slipping test, in each gender separately and combined; however, the differences did not prove significance. This result is in line with previous data from our group suggesting that the sensitivity of the slipping test might not be suitable for assessment of motor balance and co-ordination differences following neonatal HI (Rocha-Ferreira et al., 2018). At P28 the neonatal hippocampus is not fully developed (Semple 


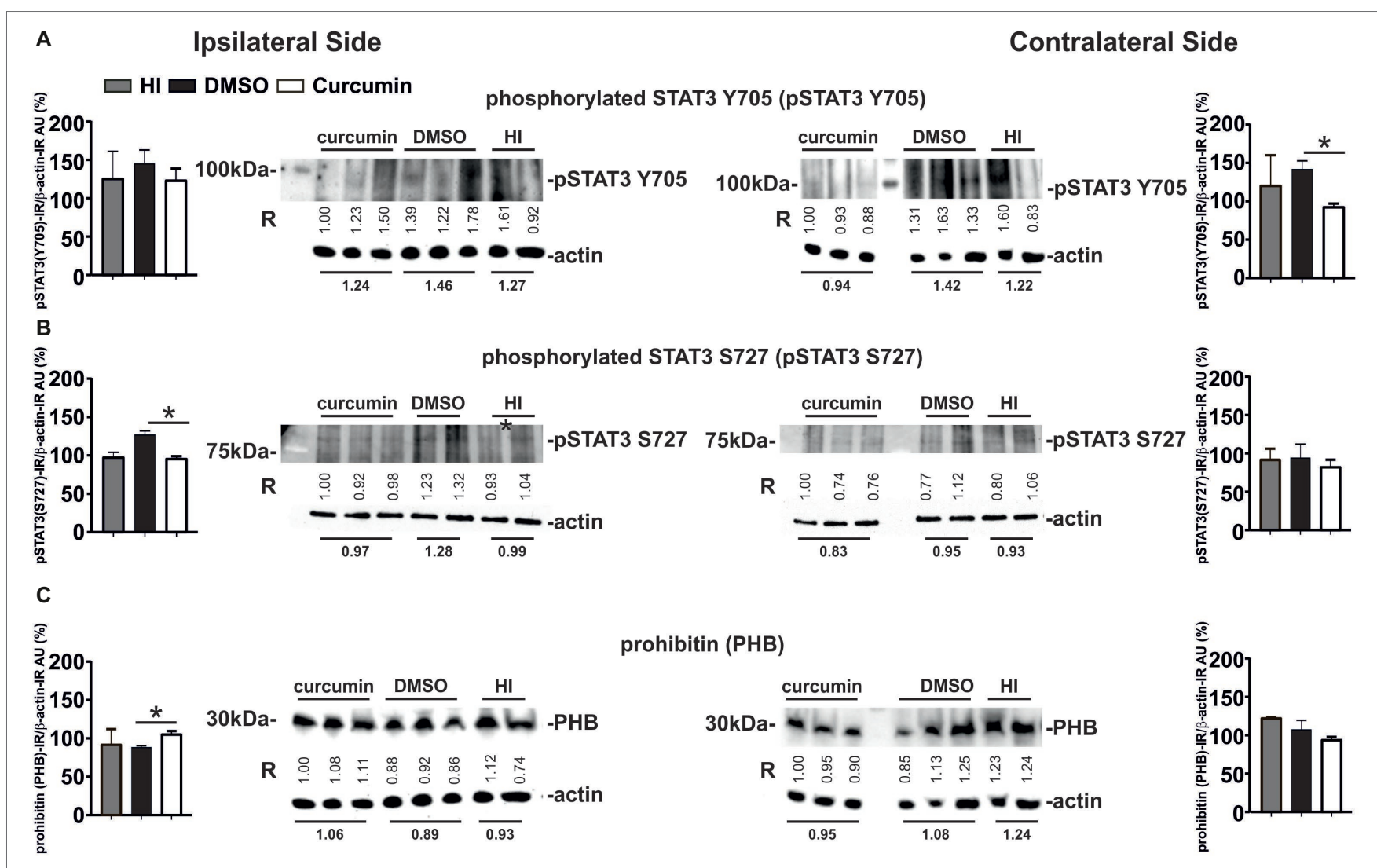

FIGURE 7 | Intraperitoneal injection of curcumin (200 $\mathrm{\mu g} / \mathrm{g}$ BW) in P7 mice immediately post-HI decreases phosphorylated STAT3 Y705 and S727 in hippocampus, while increasing ipsilateral PHB protein levels. (A) Western Blots for pSTAT3 (Y705) in ipsilateral and contralateral hippocampus from animals with HI, DMSO or $200 \mu \mathrm{g} / \mathrm{g}$ curcumin treatment and $1 \mathrm{~h}$ recovery. $\beta$-actin protein levels served as control. Note the increase of STAT3 Y705 ipsi- and contralaterally in the DMSOtreated group (19 and 16\%, respectively) when compared to HI. Curcumin treatment decreases STAT3 Y705 protein levels ipsi- and contralaterally by 3 and $22 \%$, respectively when compared to $\mathrm{HI}$, and by 15 and $33 \%(p=0.04)$ when compared to DMSO treatment. (B) Western Blots for pSTAT3 (S727) in ipsilateral and contralateral hippocampus from animals with $\mathrm{HI}$, DMSO or $200 \mathrm{\mu g} / \mathrm{g}$ curcumin treatment and $1 \mathrm{~h}$ recovery. $\beta$-actin protein levels served as control. Note the increase of STAT3 S727 ipsi- and contralaterally in the DMSO-treated group (29 and 2\%, respectively) when compared to HI. Curcumin treatment decreases STAT3 Y705 protein levels ipsi- and contralaterally by 3 and 10\%, respectively when compared to $\mathrm{HI}$, and by $25 \%(p=0.009)$ and $12 \%$ when compared to DMSO treatment. (C) Western Blots for PHB in ipsilateral and contralateral hippocampus from animals with $\mathrm{HI}$, DMSO or $200 \mu \mathrm{g} / \mathrm{g}$ curcumin treatment and $1 \mathrm{~h}$ recovery. $\beta$-actin protein levels served as control. Note the ipsilateral 13 and $19 \%(p=0.0026)$ increase of PHB protein levels in the curcumin treated animals compared to HI and DMSO, respectively. Interestingly curcumin treatment results in contralateral 23 and $12 \%$ decrease of PHB protein levels compared to $\mathrm{HI}$ and DMSO littermates, respectively. PHB levels in DMSO-treated littermates were reduced by $4 \%$ in the ipsilateral and by $13 \%$ in the contralateral side compared to littermate $\mathrm{HI}$ animals. The ratio between pSTAT3 or PHB and $\beta$-actin immunoreactivity is shown in arbitrary units (AU) set as $100 \%$ for the first line of curcumin treated animals on each of the blots. "R" represents relative densitometry compared to $\beta$-actin, which was used as the internal loading control.

et al., 2013) and it is possible that the damage is not reflected through motor and co-ordination challenges, thus assessments at later time points would be more indicative. Additionally, some data suggest that unilateral hippocampal damage is compensated by the contralateral healthy hippocampus, thus, obscuring behavioral outcome (Warburton et al., 2001; Jenkins et al., 2006). Therefore, more robust cognitive behavioral tests assessing short- and longterm memory post $\mathrm{HI}$ might prove more effective.

Curcumin is also a hormetin, thus demonstrating stimulatory properties at low doses and inhibitory ones at high doses. Therefore curcumin acts in a dose-dependent manner with lower doses being possibly more effective than higher ones, thus indicative of a hormetic response (Moghaddam et al., 2018). Dose response studies in vivo are essential for establishing whether a dietary factor affects organisms and cells via a hormetic mechanism. We tested four different doses of curcumin, i.e.,
$200,50,22$, and $10 \mu \mathrm{g} / \mathrm{g}$, and determined $50 \mu \mathrm{g} / \mathrm{g}$ as the minimal neuroprotective dose in neonatal HI brain damage (Figure 4, Supplementary Figure S1). As our data show no effect of the low doses $(22$ and $10 \mu \mathrm{g} / \mathrm{g})$, and a protective effect at 50 and $200 \mu \mathrm{g} / \mathrm{g}$, at the tested doses curcumin does not seem to exert hormetic properties when applied post injury in neonatal HI.

Curcumin pre-treatment with $100 \mu \mathrm{g} / \mathrm{g}$ BW significantly attenuates hypoxia-induced cerebral transvascular leakage, with concomitant downregulation in the expression of brain $\mathrm{NFKB}$ levels (Himadri et al., 2010). However, our results using the same pre-treatment curcumin dose but in the model of neonatal $\mathrm{HI}$, increased the levels of brain damage markers (Figure 1) suggesting detrimental effect of the compound if present at the time of the insult. A possible explanation for the negative effect of curcumin in pre-treatment for $\mathrm{HI}$ can be attributed to the well-established anti-oxidant properties of the compound 
(Marchiani et al., 2014; Akter et al., 2019). Thus, when present during the $\mathrm{HI}$ insult in the neonate, curcumin possibly additionally reduces blood oxygen levels compared to the DMSO-treated littermates, and actually proves damaging. Interestingly, all damage markers are increased in hippocampus of the curcumin treated group, which might be due to the high metabolic rate and oxygen demand of that region (Rocha-Ferreira and Hristova, 2016). Another possibility for the contradiction in the pre-treatment results is the different developmental stage: in adults with hypoxiainduced cerebral transvascular leakage all compensatory and antioxidant pathways are well established, compared to the underdeveloped neonatal brain subjected to HI injury.

Investigating the mechanisms behind the short-term neuroprotective effects of curcumin post-treatment following neonatal HI, we explored whether the treatment had an effect on total and cell-specific proliferation. HI brain damage naturally boosts cell proliferation in the neonatal brain (Rocha-Ferreira and Hristova, 2016), however due to the magnitude of the damage, that proliferation is insufficient to provide the necessary repair. Curcumin is reported to stimulate cell differentiation (Gu et al., 2012; Mujoo et al., 2012; Chen et al., 2014). Therefore, we investigated whether the neuroprotective effects of curcumin in neonatal $\mathrm{HI}$ could be attributed to an increase of the levels of cell proliferation thus stimulating repair. In line with previous studies (Rocha-Ferreira and Hristova, 2016), we observed some but not significant HI-induced increase in cell proliferation compared to naïve animals, and no significant effect of curcumin treatment. Thus, we conclude that at $48 \mathrm{~h}$ following neonatal HI brain damage the neuroprotection provided by curcumin post-treatment is not a result of effects on cell proliferation.

The inhibitory effect of curcumin on the transcription properties of STAT3, a downstream target of IL6 as a main participant in $\mathrm{HI}$ brain damage (Rocha-Ferreira and Hristova, 2016), is well documented (Alexandrow et al., 2012; Devi et al., 2015; Patel et al., 2019). Previous work from our group demonstrates that phosphorylated STAT3 Y705 is bilaterally upregulated in cortex and hippocampus following neonatal HI brain damage, and its inhibition results in neuroprotection (Hristova et al., 2016). Interestingly, at $1 \mathrm{~h}$ post-HI, we observed only contralateral significant reduction in the protein levels of phosphorylated STAT3 Y705 in hippocampus of curcumin treated animals (Figure 7A) compared to DMSO-treated littermates but not to HI controls. Similar trend is also observed in the ipsilateral hippocampus; however, the differences were not significant. Detrimental effects of DMSO treatment have previously been reported (Galvao et al., 2014) and our results suggest that DMSO treatment has a harmful effect which is counteracted by curcumin application (Figure 7A). STAT3 is an important regulator in astrocyte differentiation (Hong and Song, 2014). Although, at $1 \mathrm{~h}$ post-HI, we observed only contralateral reduction of pSTAT3 Y705 compared to DMSOtreated animals, we registered bilateral reduction of astroglial activation following post-treatment with $200 \mu \mathrm{g} / \mathrm{g}$ curcumin after neonatal HI (Figures 2G,M).

Curcumin treatment in a mouse model of stroke, reduced microglial activation and the infraction volume of the injured area compared to untreated controls (Liu et al., 2017). In the same study, curcumin treatment reduced microglial gene expression of pro-inflammatory and oxidative stress markers such as TNFa, IL12 and iNOS, and in vitro incubation of LPS- or IFN $\gamma$ activated microglia with curcumin reduced the release of pro-inflammatory cytokines (Liu et al., 2017). Our data is in line with these findings suggesting that the observed reduction in tissue loss, cell death and glial response could rely on the ability of curcumin to resolve inflammation via downregulation of pro-inflammatory cytokines.

A small pool of STAT3 has recently been discovered in mitochondria (mito-STAT3), regulating mitochondrial electron transport chain, affecting mitochondrial metabolism and cellular function (Yang and Rincon, 2016). Mito-STAT3 activation is mediated by Ser727 phosphorylation and has been shown to be crucial in immunological effector function and in cancer progression. Mito-Stat3 suppresses ROS formation during cancer genesis, suggesting that targeting Ser727 phosphorylation and mitoSTAT3 has strong potential in treating cancer. This effect of mitoSTAT3 could be critical in neonatal HI as the formation of ROS is a major factor causing cell death during the secondary energy failure (Rocha-Ferreira and Hristova, 2016), although the mechanisms of action might not be necessarily the same as in cancer genesis. Our data demonstrates ipsilateral reduction of phosphorylated STAT3 S727 in hippocampus following curcumin treatment post-HI compared to DMSO-treated animals but not to HI controls (Figure 7B) at $1 \mathrm{~h}$ post-HI. This suggests a detrimental role of DMSO-treatment in neonatal $\mathrm{HI}$ brain damage, which is counteracted by curcumin. STAT3 regulates a metabolic function in mitochondria through STAT3 S727 phosphorylation, supporting Ras-dependent malignant transformation (Gough et al., 2009). Previous data from our lab have confirmed that global pharmacological inhibition of ERK phosphorylation is strongly neuroprotective in neonatal HI brain damage (Thei et al., 2018). Thus, as mito-STAT3 is a downstream target of Ras/ERK (Yang and Rincon, 2016) our data were in line with these previous studies and reduced phosphorylation of STAT3 S727 could be neuroprotective.

Our data demonstrates bilateral increase of phosphorylated STAT3 Y705 and S727 levels in the DMSO-treated animals compared to $\mathrm{HI}$ controls at $1 \mathrm{~h}$ post-HI, which is in line with data from other groups reporting that low doses of DMSO induce caspase-3 independent neuronal death that involves apoptosis-inducing factor (AIF) translocation from mitochondria to the nucleus and poly-(ADP-ribose)-polymerase (PARP) activation (Galvao et al., 2014). Thus our results showing decrease of only $3 \%$ ipsi- and $22 \%$ contralaterally for STAT3 Y705, and of 3\% ipsi- and 10\% contralaterally for STAT3 S727 in curcumin treated compared to HI control animals is likely a result of necessity for curcumin treatment to compensate for the detrimental effects of DMSO and provide further neuroprotection.

PHB is a mitochondrial protein which has emerged as an important modulator of neuronal survival in different injury models (Zhou et al., 2012; Hernando-Rodríguez and Artal-Sanz, 2018). PHB localizes to the inner membrane of mitochondria acting as a chaperone protein, but is also found in the nucleus, where it negatively regulates transcription. PHB is significantly increased in the whole cell and markedly decreased in the nuclear 
matrix after curcumin treatment of $\mathrm{HaCaT}$ cells (Yang et al, 2014). Overexpression of PHB has been proven neuroprotective in a mouse model of middle cerebral artery occlusion (Kahl et al., 2018). Knocking down PHB by siRNA partly increased the apoptosis level of the neuronal cell line PC12 stimulated by $\mathrm{H}_{2} \mathrm{O}_{2}$ (Xu et al., 2014). Our results at $1 \mathrm{~h}$ post-HI show ipsilateral increase of the protein levels of PHB in hippocampus following curcumin treatment post-HI (Figure 7C). This is in line with the effects observed by other groups, generally associating increased PHB expression with decrease of cell death, amelioration of mitochondrial dysfunction and neuroprotection. However, it is unclear whether, similarly to STAT3 Y705 (Hristova et al., 2016) PHB plays a different role in different cell types involved in neonatal HI, and whether its expression is time-dependent.

In conclusion, our data support a dose-dependent neuroprotection provided by immediate and delayed treatment with curcumin following neonatal $\mathrm{HI}$ injury. The precise mechanism of this protection is unclear; however, our results show effects of curcumin on oxidative stress and myelination, inflammation and transcription (STAT3 Y705) and mitochondrial dysfunction (STAT3 S727 and PHB). This makes curcumin an attractive therapeutic candidate in neonatal $\mathrm{HI}$ induced brain damage.

Although phase I clinical trials have shown that curcumin is safe even at high doses (12 g/day), its future use and clinical application is limited as a result of its poor bioavailability due to poor absorption, rapid metabolism, and rapid systemic elimination (Anand et al., 2007; Gupta et al., 2013). Therefore, future experiments should focus on increasing curcumin bioavailability and solubility with the development of aqueous solutions for clinical application.

\section{ETHICS STATEMENT}

All animal experiments and care protocols were carried out according to the UK Animals (Scientific Procedures) Act 1986 and approved by the Home Office (PPL70/8784). The ARRIVE guidelines were followed. All experiments involved postnatal day 7 C57/Bl6 mice (P7) bred in house.

\section{AUTHOR CONTRIBUTIONS}

ER-F, CS, and SL contributed to the collection and processing of data, writing and editing the manuscript. SB, TF, MHa, ICR, CA,

\section{REFERENCES}

Ahmad, M. Z., Alkahtani, S. A., Akhter, S., Ahmad, F. J., Ahmad, J., Akhtar, M. S., et al. (2016). Progress in nanotechnology-based drug carrier in designing of curcumin nanomedicines for cancer therapy: current state-of-the-art. J. Drug Target. 24, 273-293. doi: 10.3109/1061186X.2015.1055570

Akter, J., Hossain, M. A., Takara, K., Islam, M. Z., and Hou, D.-X. (2019). Antioxidant activity of different species and varieties of turmeric (Curcuma spp): isolation of active compounds. Comp. Biochem. Physiol. Part C Toxicol. Pharmacol. 215, 9-17. doi: 10.1016/j.cbpc.2018.09.002

Alexandrow, M. G., Song, L. J., Altiok, S., Gray, J., Haura, E. B., and Kumar, N. B. (2012). Curcumin. Eur. J. Cancer Prev. 21, 407-412. doi: 10.1097/CEJ.0b013e32834ef194
TK, and $\mathrm{BM}$ assisted with the collection and processing of data. DH contributed to the collection and processing of data, editing the manuscript. MHr contributed to the design of the study, collection and processing of data, writing and editing the manuscript.

\section{FUNDING}

This work was supported by the BBSRC LIDo programme BB/M009513/1.

\section{SUPPLEMENTARY MATERIAL}

The Supplementary Material for this article can be found online at: https://www.frontiersin.org/articles/10.3389/fphys.2019.01351/ full\#supplementary-material

SUPPLEMENTARY FIGURE S1 | Intraperitoneal injection of curcumin in P7 mice post-HI significantly reduces in a dose dependent manner ipsilateral brain tissue volume loss, TUNEL+ cell death and ipsilateral and contralateral reactive astrogliosis. (A) Intraperitoneal injection of $50 \mathrm{\mu g} / \mathrm{g}$ BW curcumin decreased ipsilateral volume loss compared to DMSO treated littermates, with significant individual decrease (t-test) in cortex $(p=0.018)$, hippocampus $(p=0.025)$ and overall $(p=0.0004)$. Mixed Linear Model treating region as a repeated measure revealed $p=0.0001$. (B,C) Intraperitoneal injection of $22 \mu \mathrm{g} / \mathrm{g}$ (B) and $10 \mu \mathrm{g} / \mathrm{g}$ (C) curcumin did not have an effect on volume tissue loss compared to DMSO treated littermates. (D) Intraperitoneal injection of $50 \mu \mathrm{g} / \mathrm{g}$ curcumin decreased ipsilateral TUNEL+ cell death compared to DMSO treated littermates, with significant individual decrease ( $t$-test) in hippocampus $(p=0.007)$ and external capsule $(p=0.0001)$. Mixed Linear Model treating region as a repeated measure revealed $p=0.016$. (E,F) Intraperitoneal injection of $22 \mu \mathrm{g} / \mathrm{g}$ (E) and $10 \mu \mathrm{g} / \mathrm{g}$ (F) curcumin did not have an effect on ipsilateral TUNEL+ cell death compared to DMSO treated littermates. (G) Intraperitoneal injection of $50 \mu \mathrm{g} / \mathrm{g}$ curcumin decreased ipsilateral reactive astrogliosis assessed though OLV of GFAP immunoreactivity compared to DMSO treated littermates, with significant individual decrease ( $t$-test) in pyriform cortex $(p=0.025)$, hippocampus $(p=0.033)$,

thalamus $(p=0.030)$ and external capsule $(p=0.031)$. Mixed Linear Model treating region as a repeated measure revealed $p=0.014$. $(\mathbf{H}, \mathbf{I})$ Intraperitoneal injection of $22 \mu \mathrm{g} / \mathrm{g} \mathbf{( H )}$ and $10 \mu \mathrm{g} / \mathrm{g}$ (I) curcumin did not have an effect on ipsilateral TUNEL+ cell death compared to DMSO treated littermates. (J) Intraperitoneal injection of $50 \mu \mathrm{g} / \mathrm{g}$ curcumin reduced contralateral reactive astrogliosis compared to DMSO treated littermates, with significant individual decrease ( $t$-test) in pyriform cortex $(p=0.009)$. Mixed Linear Model treating region as a repeated measure revealed $p=0.005$. (K, L) Intraperitoneal injection of $22 \mu \mathrm{g} / \mathrm{g} \mathbf{( H )}$ and $10 \mu \mathrm{g} / \mathrm{g}$ (I) curcumin did not have an effect on contralateral astrogliosis compared to DMSO treated littermates. (A, curcumin $50 \mu \mathrm{g} / \mathrm{g} n=8$, DMSO $n=6$, B, curcumin $22 \mu \mathrm{g} / \mathrm{g} n=12$, DMSO $n=10, \mathbf{C}$, curcumin $10 \mu \mathrm{g} / \mathrm{g} n=11$, DMSO $n=11)\left({ }^{*} p<0.05\right)$.

Abbreviations: CTX, cerebral cortex; EC, external capsule; HIP, hippocampus; PYR, pyriform cortex; STR, striatum; THAL, thalamus. 
Chen, F., Wang, H., Xiang, X., Yuan, J., Chu, W., Xue, X., et al. (2014). Curcumin increased the differentiation rate of neurons in neural stem cells via wnt signaling in vitro study. J. Surg. Res. 192, 298-304. doi: 10.1016/j.jss.2014.06.026

Clarkson, J., and Herbison, A. E. (2016). Hypothalamic control of the male neonatal testosterone surge. Philos. Trans. R. Soc. Lond. Ser. B Biol. Sci. 371:20150115. Available at: http://rstb.royalsocietypublishing.org/content/371/1688/20150115 (Accessed September 5, 2017).

Cui, X., Song, H., and Su, J. (2017). Curcumin attenuates hypoxic-ischemic brain injury in neonatal rats through induction of nuclear factor erythroid-2related factor 2 and heme oxygenase-1. Exp. Ther. Med. 14, 1512-1518. doi: 10.3892/etm.2017.4683

Devi, Y. S., DeVine, M., DeKuiper, J., Ferguson, S., and Fazleabas, A. T. (2015). Inhibition of IL-6 signaling pathway by curcumin in uterine decidual cells. PLoS One 10:e0125627. doi: 10.1371/journal.pone.0125627

Dhillon, N., Aggarwal, B. B., Newman, R. A., Wolff, R. A., Kunnumakkara, A. B., Abbruzzese, J. L., et al. (2008). Phase II trial of curcumin in patients with advanced pancreatic cancer. Clin. Cancer Res. 14, 4491-4499. doi: 10.1158/ 1078-0432.CCR-08-0024

Galvao, J., Davis, B., Tilley, M., Normando, E., Duchen, M. R., and Cordeiro, M. F. (2014). Unexpected low-dose toxicity of the universal solvent DMSO. FASEB J. 28, 1317-1330. doi: 10.1096/fj.13-235440

Gluckman, P. D., Wyatt, J. S., Azzopardi, D., Ballard, R., Edwards, A. D., Ferriero, D. M., et al. (2005). Selective head cooling with mild systemic hypothermia after neonatal encephalopathy: multicentre randomised trial. Lancet 365, 663-670. doi: 10.1016/S0140-6736(05)17946-X

Gobinath, A. R., Choleris, E., and Galea, L. A. M. (2017). Sex, hormones, and genotype interact to influence psychiatric disease, treatment, and behavioral research. J. Neurosci. Res. 95, 50-64. doi: 10.1002/jnr.23872

Gough, D. J., Corlett, A., Schlessinger, K., Wegrzyn, J., Larner, A. C., and Levy, D. E. (2009). Mitochondrial STAT3 supports Ras-dependent oncogenic transformation. Science 324, 1713-1716. doi: 10.1126/science.1171721

Gu, Q., Cai, Y., Huang, C., Shi, Q., and Yang, H. (2012). Curcumin increases rat mesenchymal stem cell osteoblast differentiation but inhibits adipocyte differentiation. Pharmacogn. Mag. 8, 202-208. doi: 10.4103/0973-1296.99285

Gupta, S. C., Patchva, S., and Aggarwal, B. B. (2013). Therapeutic roles of curcumin: lessons learned from clinical trials. AAPS J. 15, 195-218. doi: 10.1208/s12248-012-9432-8

He, H., Luo, Y., Qiao, Y., Zhang, Z., Yin, D., Yao, J., et al. (2018). Curcumin attenuates doxorubicin-induced cardiotoxicity via suppressing oxidative stress and preventing mitochondrial dysfunction mediated by 14-3-3 $\gamma$. Food Funct. 9, 4404-4418. doi: 10.1039/C8FO00466H

Hernando-Rodríguez, B., and Artal-Sanz, M. (2018). Mitochondrial quality control mechanisms and the PHB (Prohibitin) complex. Cell 7:238. doi: $10.3390 /$ cells7120238

Himadri, P., Kumari, S. S., Chitharanjan, M., and Dhananjay, S. (2010). Role of oxidative stress and inflammation in hypoxia-induced cerebral edema: a molecular approach. High Alt. Med. Biol. 11, 231-244. doi: 10.1089/ham.2009.1057

Hong, S., and Song, M.-R. (2014). STAT3 but not STAT1 is required for astrocyte differentiation. PLoS One 9:e86851. doi: 10.1371/journal.pone.0086851

Hope, P. L., Costello, A. M., Cady, E. B., Delpy, D. T., Tofts, P. S., Chu, A., et al. (1984). Cerebral energy metabolism studied with phosphorus NMR spectroscopy in normal and birth-asphyxiated infants. Lancet 2, 366-370. Available at: http:// www.ncbi.nlm.nih.gov/pubmed/6147452 (Accessed January 12, 2016).

Hristova, M., Cuthill, D., Zbarsky, V., Acosta-Saltos, A., Wallace, A., Blight, K., et al. (2010). Activation and deactivation of periventricular white matter phagocytes during postnatal mouse development. Glia 58, 11-28. doi: 10.1002/glia.20896

Hristova, M., Rocha-Ferreira, E., Fontana, X., Thei, L., Buckle, R., Christou, M., et al. (2016). Inhibition of signal transducer and activator of transcription 3 (STAT3) reduces neonatal hypoxic-ischaemic brain damage. J. Neurochem. 136, 981-994. doi: 10.1111/jnc.13490

Hsiao, Y.-T., Kuo, C.-L., Chueh, F.-S., Liu, K.-C., Bau, D.-T., and Chung, J.-G. (2018). Curcuminoids induce reactive oxygen species and autophagy to enhance apoptosis in human oral cancer cells. Am. J. Chin. Med. 46, 1145-1168. doi: 10.1142/S0192415X1850060X

Jaisin, Y., Thampithak, A., Meesarapee, B., Ratanachamnong, P., Suksamrarn, A., Phivthong-ngam, L., et al. (2011). Curcumin I protects the dopaminergic cell line SH-SY5Y from 6-hydroxydopamine-induced neurotoxicity through attenuation of p53-mediated apoptosis. Neurosci. Lett. 489, 192-196. doi: 10.1016/j.neulet.2010.12.014
Jenkins, T. A., Amin, E., Brown, M. W., and Aggleton, J. P. (2006). Changes in immediate early gene expression in the rat brain after unilateral lesions of the hippocampus. Neuroscience 137, 747-759. doi: 10.1016/J. NEUROSCIENCE.2005.09.034

Johnston, M. V., Nakajima, W., and Hagberg, H. (2002). Mechanisms of hypoxic neurodegeneration in the developing brain. Neuroscientist 8, 212-220. Available at: http://www.ncbi.nlm.nih.gov/pubmed/12061501 (Accessed October 23, 2015).

Joseph, A., Wood, T., Chen, C.-C., Corry, K., Snyder, J. M., Juul, S. E., et al. (2018). Curcumin-loaded polymeric nanoparticles for neuroprotection in neonatal rats with hypoxic-ischemic encephalopathy. Nano Res. 11, 5670-5688. doi: 10.1007/s12274-018-2104-y

Kahl, A., Anderson, C. J., Qian, L., Voss, H., Manfredi, G., Iadecola, C., et al. (2018). Neuronal expression of the mitochondrial protein prohibitin confers profound neuroprotection in a mouse model of focal cerebral ischemia J. Cereb. Blood Flow Metab. 38, 1010-1020. doi: 10.1177/0271678X17720371

Kaushal, P., Mehra, R. D., and Dhar, P. (2014). Curcumin induced up-regulation of myelin basic protein (MBP) ameliorates sodium arsenite induced neurotoxicity in developing rat cerebellum. J. Anat. Soc. India 63, 3-11. doi: 10.1016/J.JASI.2014.04.001

Kendall, G. S., Hristova, M., Zbarsky, V., Clements, A., Peebles, D. M., Robertson, N. J., et al. (2012). Distribution of $\mathrm{pH}$ changes in mouse neonatal hypoxic-ischaemic insult. Dev. Neurosci. 33, 505-518. doi: 10.1159/000333850

Kendall, G. S., Robertson, N. J., Iwata, O., Peebles, D., and Raivich, G. (2006). $\mathrm{N}$-methyl-isobutyl-amiloride ameliorates brain injury when commenced before hypoxia ischemia in neonatal mice. Pediatr. Res. 59, 227-231. doi: 10.1203/01. pdr.0000196805.68082.22

Kim, J. E., Kim, A. R., Chung, H. Y., Han, S. Y., Kim, B. S., and Choi, J. S. (2003). In vitro peroxynitrite scavenging activity of diarylheptanoids from Curcuma longa. Phyther. Res. 17, 481-484. doi: 10.1002/ptr.1179

Lange, S., Rocha-Ferreira, E., Thei, L., Mawjee, P., Bennett, K., Thompson, P. R., et al. (2014). Peptidylarginine deiminases: novel drug targets for prevention of neuronal damage following hypoxic ischemic insult $(\mathrm{HI})$ in neonates. J. Neurochem. 130, 555-562. doi: 10.1111/jnc.12744

Lawn, J. E., Cousens, S., and Zupan, J. (2005). 4 million neonatal deaths: when? Where? Why? Lancet 365, 891-900. doi: 10.1016/S0140-6736(05)71048-5

Li, Y., Powers, C., Jiang, N., and Chopp, M. (1998). Intact, injured, necrotic and apoptotic cells after focal cerebral ischemia in the rat. J. Neurol. Sci. 156, 119-132. Available at: http://www.ncbi.nlm.nih.gov/pubmed/9588846 (Accessed October 31, 2015).

Liu, Y., and Hong, X.-Q. (2006). Effect of three different curcumin pigmens on the prdiferation of vascular smooth muscle cells by ox-LDL and the expression of LDL-R. Zhongguo Zhong Yao Za Zhi 31, 500-503.

Liu, Z., Ran, Y., Huang, S., Wen, S., Zhang, W., Liu, X., et al. (2017). Curcumin protects against ischemic stroke by titrating microglia/macrophage polarization. Front. Aging Neurosci. 9:233. doi: 10.3389/fnagi.2017.00233

Lundgren, C., Brudin, L., Wanby, A.-S., and Blomberg, M. (2018). Ante- and intrapartum risk factors for neonatal hypoxic ischemic encephalopathy. J. Matern. Neonatal Med. 31, 1595-1601. doi: 10.1080/14767058.2017.1321628

Luong, T. N., Carlisle, H. J., Southwell, A., and Patterson, P. H. (2011). Assessment of motor balance and coordination in mice using the balance beam. J. Vis. Exp. 49. doi: $10.3791 / 2376$

Maheshwari, R. K., Singh, A. K., Gaddipati, J., and Srimal, R. C. (2006). Multiple biological activities of curcumin: a short review. Life Sci. 78, 2081-2087. doi: 10.1016/j.lfs.2005.12.007

Marchiani, A., Rozzo, C., Fadda, A., Delogu, G., and Ruzza, P. (2014). Curcumin and curcumin-like molecules: from spice to drugs. Curr. Med. Chem. 21, 204-222. Available at: http://www.ncbi.nlm.nih.gov/pubmed/23590716 (Accessed January 24, 2019).

Mayoral, S. R., Omar, G., and Penn, A. A. (2009). Sex differences in a hypoxia model of preterm brain damage. Pediatr. Res. 66, 248-253. doi: 10.1203/ PDR.0b013e3181b1bc34

Merkwirth, C., Martinelli, P., Korwitz, A., Morbin, M., Brönneke, H. S., Jordan, S. D., et al. (2012). Loss of prohibitin membrane scaffolds impairs mitochondrial architecture and leads to tau hyperphosphorylation and neurodegeneration. PLoS Genet. 8:e1003021. doi: 10.1371/journal.pgen.1003021

Moghadamtousi, S. Z., Kadir, H. A., Hassandarvish, P., Tajik, H., Abubakar, S., Zandi, K., et al. (2014). A review on antibacterial, antiviral, and antifungal activity of curcumin. Biomed. Res. Int. 2014:186864. doi: $10.1155 / 2014 / 186864$ 
Moghaddam, N. S. A., Oskouie, M. N., Butler, A. E., Petit, P. X., Barreto, G. E., and Sahebkar, A. (2018). Hormetic effects of curcumin: what is the evidence? J. Cell. Physiol. 2014, 10060-10071. doi: 10.1002/jcp.27880

Möller, J. C., Klein, M. A., Haas, S., Jones, L. L., Kreutzberg, G. W., and Raivich, G. (1996). Regulation of thrombospondin in the regenerating mouse facial motor nucleus. Glia 17, -121, 32. doi: 10.1002/(SICI)1098-1136(199606) 17:2\&lt;121::AID-GLIA4\&gt;3.0.CO;2-5

Momekova, D., Ugrinova, I., Slavkova, M., Momekov, G., Grancharov, G., Gancheva, V., et al. (2018). Superior proapoptotic activity of curcuminloaded mixed block copolymer micelles with mitochondrial targeting properties. Biomater. Sci. 6, 3309-3317. doi: 10.1039/C8BM00644J

Mujoo, K., Nikonoff, L. E., Sharin, V. G., Bryan, N. S., Kots, A. Y., and Murad, F. (2012). Curcumin induces differentiation of embryonic stem cells through possible modulation of nitric oxide-cyclic GMP pathway. Protein Cell 3, 535-544. doi: 10.1007/s13238-012-2053-2

Mutsuga, M., Chambers, J. K., Uchida, K., Tei, M., Makibuchi, T., Mizorogi, T., et al. (2012). Binding of curcumin to senile plaques and cerebral amyloid angiopathy in the aged brain of various animals and to neurofibrillary tangles in Alzheimer's brain. J. Vet. Med. Sci. 74, 51-57. doi: 10.1292/ jvms.11-0307

Mythri, R. B., Harish, G., Dubey, S. K., Misra, K., and Srinivas Bharath, M. M. (2011). Glutamoyl diester of the dietary polyphenol curcumin offers improved protection against peroxynitrite-mediated nitrosative stress and damage of brain mitochondria in vitro: implications for Parkinson's disease. Mol. Cell. Biochem. 347, 135-143. doi: 10.1007/s11010-010-0621-4

Naksuriya, O., Okonogi, S., Schiffelers, R. M., and Hennink, W. E. (2014). Curcumin nanoformulations: a review of pharmaceutical properties and preclinical studies and clinical data related to cancer treatment. Biomaterials 35, 3365-3383. doi: 10.1016/j.biomaterials.2013.12.090

Naserzadeh, P., Hafez, A. A., Abdorahim, M., Abdollahifar, M. A., Shabani, R., Peirovi, H., et al. (2018). Curcumin loading potentiates the neuroprotective efficacy of $\mathrm{Fe}_{3} \mathrm{O}_{4}$ magnetic nanoparticles in cerebellum cells of schizophrenic rats. Biomed. Pharmacother. 108, 1244-1252. doi: 10.1016/j.biopha.2018.09.106

Nishiyama, T., Mae, T., Kishida, H., Tsukagawa, M., Mimaki, Y., Kuroda, M., et al. (2005). Curcuminoids and Sesquiterpenoids in turmeric (Curcuma longa L.) suppress an increase in blood glucose level in type 2 diabetic KK-Ay mice. J. Agric. Food Chem. 53, 959-963. doi: 10.1021/jf0483873

Patel, S. S., Acharya, A., Ray, R. S., Agrawal, R., Raghuwanshi, R., and Jain, P. (2019). Cellular and molecular mechanisms of curcumin in prevention and treatment of disease. Crit. Rev. Food Sci. Nutr. 1-53. doi: 10.1080/10408398. 2018.1552244

Peng, Y.-T., Chen, P., Ouyang, R.-Y., and Song, L. (2015). Multifaceted role of prohibitin in cell survival and apoptosis. Apoptosis 20, 1135-1149. doi: 10.1007/s10495-015-1143-Z

Peng, T. I., and Greenamyre, J. T. (1998). Privileged access to mitochondria of calcium influx through N-methyl-D-aspartate receptors. Mol. Pharmacol. 53, 974-980.

Penrice, J., Lorek, A., Cady, E. B., Amess, P. N., Wylezinska, M., Cooper, C. E., et al. (1997). Proton magnetic resonance spectroscopy of the brain during acute hypoxia-ischemia and delayed cerebral energy failure in the newborn piglet. Pediatr. Res. 41, 795-802. doi: 10.1203/00006450-199706000-00001

Pescosolido, N., Giannotti, R., Plateroti, A., Pascarella, A., and Nebbioso, M. (2013). Curcumin: therapeutical potential in ophthalmology. Planta Med. 80, 249-254. doi: 10.1055/s-0033-1351074

Priyadarsini, K., and Indira, K. (2014). The chemistry of curcumin: from extraction to therapeutic agent. Molecules 19, 20091-20112. doi: 10.3390/ molecules191220091

Puka-Sundvall, M., Hallin, U., Zhu, C., and Wang, X. (2000). NMDA blockade attenuates caspase-3 activation and DNA fragmentation after neonatal hypoxia-ischemia. Dev. Neurosci. 11, 2833-2836.

Raivich, G., Bohatschek, M., Kloss, C. U. A., Werner, A., Jones, L. L., and Kreutzberg, G. W. (1999). Neuroglial activation repertoire in the injured brain: graded response, molecular mechanisms and cues to physiological function. Brain Res. Rev. 30, 77-105. doi: 10.1016/S0165-0173(99)00007-7

Ray, B., Bisht, S., Maitra, A., Maitra, A., and Lahiri, D. K. (2011). Neuroprotective and neurorescue effects of a novel polymeric nanoparticle formulation of curcumin (NanoCurc ${ }^{\mathrm{Tx}}$ ) in the neuronal cell culture and animal model: implications for Alzheimer's disease. J. Alzheimers Dis. 23, 61-77. doi: 10.3233/ JAD-2010-101374

Rocha-Ferreira, E., and Hristova, M. (2016). Plasticity in the neonatal brain following hypoxic-ischaemic injury. Neural Plast. 2016, 1-16. doi: 10.1155/2016/4901014

Rocha-Ferreira, E., Phillips, E., Francesch-Domenech, E., Thei, L., Peebles, D. M., Raivich, G., et al. (2015). The role of different strain backgrounds in bacterial endotoxin-mediated sensitization to neonatal hypoxic-ischemic brain damage. Neuroscience 311, 292-307. doi: 10.1016/j.neuroscience. 2015.10.035

Rocha-Ferreira, E., Vincent, A., Bright, S., Peebles, D. M., and Hristova, M. (2018). The duration of hypothermia affects short-term neuroprotection in a mouse model of neonatal hypoxic ischaemic injury. PLoS One 13:e0199890. doi: 10.1371/journal.pone.0199890

Sanders, R. D., Manning, H. J., Robertson, N. J., Ma, D., Edwards, A. D., Hagberg, H., et al. (2010). Preconditioning and postinsult therapies for perinatal hypoxic-ischemic injury at term. Anesthesiology 113, 233-249. doi: 10.1097/ALN.0b013e3181dc1b84

Sandur, S. K., Pandey, M. K., Sung, B., Ahn, K. S., Murakami, A., Sethi, G., et al. (2007). Curcumin, demethoxycurcumin, bisdemethoxycurcumin, tetrahydrocurcumin and turmerones differentially regulate anti-inflammatory and anti-proliferative responses through a ROS-independent mechanism. Carcinogenesis 28, 1765-1773. doi: 10.1093/carcin/bgm123

Semple, B. D., Blomgren, K., Gimlin, K., Ferriero, D. M., and Noble-Haeusslein, L. J. (2013). Brain development in rodents and humans: identifying benchmarks of maturation and vulnerability to injury across species. Prog. Neurobiol. 106-107, 1-16. doi: 10.1016/j.pneurobio.2013.04.001

Shishodia, S., Sethi, G., and Aggarwal, B. B. (2005). Curcumin: getting back to the roots. Ann. N. Y. Acad. Sci. 1056, 206-217. doi: 10.1196/annals.1352.010

Shukla, P. K., Khanna, V. K., Ali, M. M., Khan, M. Y., and Srimal, R. C. (2008). Anti-ischemic effect of curcumin in rat brain. Neurochem. Res. 33, 1036-1043. doi: 10.1007/s11064-007-9547-y

Spagnuolo, C., Napolitano, M., Tedesco, I., Moccia, S., Milito, A., and Russo, G. L. (2016). Neuroprotective role of natural polyphenols. Curr. Top. Med. Chem. 16, 1943-1950. doi: 10.2174/1568026616666160204122449

Tham, C. L., Liew, C. Y., Lam, K. W., Mohamad, A.-S., Kim, M. K., Cheah, Y. K., et al. (2010). A synthetic curcuminoid derivative inhibits nitric oxide and proinflammatory cytokine synthesis. Eur. J. Pharmacol. 628, 247-254. doi: 10.1016/j.ejphar.2009.11.053

Thei, L., Rocha-Ferreira, E., Peebles, D., Raivich, G., and Hristova, M. (2018). Extracellular signal-regulated kinase 2 has duality in function between neuronal and astrocyte expression following neonatal hypoxic-ischaemic cerebral injury. J. Physiol. 596, 6043-6062. doi: 10.1113/JP275649

Trujillo, J., Granados-Castro, L. F., Zazueta, C., Andérica-Romero, A. C., Chirino, Y. I., and Pedraza-Chaverrí, J. (2014). Mitochondria as a target in the therapeutic properties of curcumin. Arch. Pharm. 347, 873-884. doi: 10.1002/ardp.201400266

Vannucci, R. C., and Vannucci, S. J. (1997). A model of perinatal hypoxic-ischemic brain damage. Ann. N. Y. Acad. Sci. 234-249. doi: 10.1111/j.1749-6632.1997. tb48634.x

Warburton, E. C., Baird, A., Morgan, A., Muir, J. L., and Aggleton, J. P. (2001). The conjoint importance of the hippocampus and anterior thalamic nuclei for allocentric spatial learning: evidence from a disconnection study in the rat. J. Neurosci. 21, 7323-7330. doi: 10.1523/JNEUROSCI.21-18-07323.2001

Wu, A., Ying, Z., and Gomez-Pinilla, F. (2006). Dietary curcumin counteracts the outcome of traumatic brain injury on oxidative stress, synaptic plasticity, and cognition. Exp. Neurol. 197, 309-317. doi: 10.1016/j. expneurol.2005.09.004

Xu, T., Fan, X., Tan, Y., Yue, Y., Chen, W., Gu, X., et al. (2014). Expression of PHB2 in rat brain cortex following traumatic brain injury. Int. J. Mol. Sci. 15, 3299-3318. doi: 10.3390/ijms15023299

Yang, R., and Rincon, M. (2016). Mitochondrial Stat3, the need for design thinking. Int. J. Biol. Sci. 12, 532-544. doi: 10.7150/ijbs.15153

Yang, H.-B., Song, W., Chen, L.-Y., Li, Q.-F., Shi, S.-L., Kong, H.-Y., et al. (2014). Differential expression and regulation of prohibitin during curcumininduced apoptosis of immortalized human epidermal HaCaT cells. Int. J. Mol. Med. 33, 507-514. doi: 10.3892/ijmm.2014.1621 
Yu, S., Zheng, W., Xin, N., Chi, Z.-H., Wang, N.-Q., Nie, Y.-X., et al. (2010). Curcumin prevents dopaminergic neuronal death through inhibition of the c-Jun N-terminal kinase pathway. Rejuvenation Res. 13, 55-64. doi: 10.1089/ rej.2009.0908

Zhao, J., Yu, S., Zheng, W., Feng, G., Luo, G., Wang, L., et al. (2010). Curcumin improves outcomes and attenuates focal cerebral ischemic injury via antiapoptotic mechanisms in rats. Neurochem. Res. 35, 374-379. doi: 10.1007/ s11064-009-0065-y

Zhou, P., Qian, L., D’Aurelio, M., Cho, S., Wang, G., Manfredi, G., et al. (2012). Prohibitin reduces mitochondrial free radical production and protects brain cells from different injury modalities. J. Neurosci. 32, 583-592. doi: 10.1523/ JNEUROSCI.2849-11.2012
Conflict of Interest: The authors declare that the research was conducted in the absence of any commercial or financial relationships that could be construed as a potential conflict of interest.

Copyright $\odot 2019$ Rocha-Ferreira, Sisa, Bright, Fautz, Harris, Contreras Riquelme, Agwu, Kurulday, Mistry, Hill, Lange and Hristova. This is an open-access article distributed under the terms of the Creative Commons Attribution License (CC BY). The use, distribution or reproduction in other forums is permitted, provided the original author(s) and the copyright owner(s) are credited and that the original publication in this journal is cited, in accordance with accepted academic practice. No use, distribution or reproduction is permitted which does not comply with these terms. 